\title{
CONVOLUTION AND HYPERGROUP STRUCTURES ASSOCIATED WITH A CLASS OF STURM-LIOUVILLE SYSTEMS
}

\author{
WILLIAM C. CONNETT, CLEMENS MARKETT AND ALAN L. SCHWARTZ
}

Abstract. Product formulas of the type

$$
u_{k}(\theta) u_{k}(\phi)=\int_{0}^{\pi} u_{k}(\xi) D(\xi, \theta, \phi) d \xi
$$

are obtained for the eigenfunctions of a class of second order regular and regular singular Sturm-Liouville problems on $[0, \pi]$ by using the Riemann integration method to solve a Cauchy problem for an associated hyperbolic differential equation.

When $D(\xi, \theta, \phi)$ is nonnegative (which can be guaranteed by a simple restriction on the differential operator of the Sturm-Liouville problem), it is possible to define a convolution with respect to which $M[0, \pi]$ becomes a Banach algebra with the functions $u_{k}(\xi) / u_{0}(\xi)$ as its characters. In fact this measure algebra is a Jacobi type hypergroup. It is possible to completely describe the maximal ideal space and idempotents of this measure algebra.

\section{INTRODUCTION}

A central idea in harmonic analysis in various settings has been the existence of a product, usually called convolution, for functions and measures. In particular, in the study of the harmonic analysis of orthogonal expansions, a convolution arises in a natural way and plays the same role as ordinary convolution in Fourier analysis.

In some cases, an investigation begins with a convolution algebra of measures as the primitive object upon which to build a theory; this is the case of the analysis of the objects called hypergroups which are generalizations of the convolution algebra of Borel measures on a group. One of the subjects of this paper will be the introduction of a large class of new convolution structures, many of which are new hypergroups.

A hypergroup $(H, *)$ is a Banach algebra of the Borel measures $M(H)$ on a locally compact Hausdorff space $H$ with product $*$ called convolution which

Received by the editors May 8, 1989 and, in revised form, May 10, 1990.

1980 Mathematics Subject Classification (1985 Revision). Primary 43A10; Secondary 34B25, $42 \mathrm{~A} 85,42 \mathrm{C} 10$.

Key words and phrases. Measure algebras, convolutions, hypergroups, product formulas, SturmLiouville problems, characters, eigenfunctions.

This paper was written while the second-named author was visiting the Department of Mathematics at the University of Missouri-St. Louis. He wishes to express his gratitude to that department for its hospitality. 
satisfies the following axioms: ( $\delta_{s}$ denotes the unit mass concentrated at $s$, and for $\mu \in M(H), \operatorname{supp}(\mu)$ is the support of $\mu)$.

H1. If $\mu$ and $\nu$ are probability measures, then so is $\mu * \nu$.

H2. The mapping $(\mu, \nu) \rightarrow \mu * \nu$ is continuous from $M(H) \times M(H)$ into $M(H)$ where $M(H)$ is given the weak topology with respect to $C_{c}(H)$.

H3. There is an element $e \in H$ such that $\delta_{e} * \mu=\mu * \delta_{e}=\mu$ for every $\mu \in M(H)$.

H4. There is a homeomorphic mapping $s \rightarrow s^{\vee}$ of $H$ into itself such that $s^{\vee \vee}=s$ and $e \in \operatorname{supp}\left(\delta_{s} * \delta_{t}\right)$ if and only if $t=s^{\vee}$.

H5. For $\mu, \nu \in M(H)(\mu * \nu)^{\vee}=\nu^{\vee} * \mu^{\vee}$ where $\mu^{\vee}$ is defined by

$$
\int_{H} f(s) d \mu^{\vee}(s)=\int_{H} f\left(s^{\vee}\right) d \mu(s) .
$$

H6. The mapping $(s, t) \rightarrow \operatorname{supp}\left(\delta_{s} * \delta_{t}\right)$ is continuous from $H \times H$ into the space of compact subsets of $H$ as topologized in [24].

Hypergroups generalize the convolution algebra $M(G)$ for locally compact topological groups $G$ as can be seen in the following abbreviated discussion. (For more detail see [9 and 15] and the references cited there.)

A continuous function $\phi$ is a character of $(H, *)$ if for every $s, t \in H$, $\int_{H} \phi d\left(\delta_{s} * \delta_{t}\right)=\phi(s) \phi(t) ; \phi$ is a Hermitian character if $\phi\left(s^{\vee}\right)=\overline{\phi(s)}$; the set of Hermitian characters is denoted by $H^{\wedge}$ and the Fourier-Stieltjes transform of $\mu$ is given by

$$
\mu^{\frown}(\phi)=\int_{H} \phi d \mu \quad\left(\phi \in H^{\wedge}\right) .
$$

Assume $*$ is commutative; then $(H, *)$ possesses a positive measure $m$ such that $\operatorname{supp}(m)=H$ and $m * \delta_{s}=\delta_{s} * m=m$ for every $s \in H ; m$ is called a Haar measure for $(H, *)$.

The operators $T^{s}$ defined by $T^{s} f(t)=\int_{H} f d\left(\delta_{s} * \delta_{t}\right)$ are called generalized translations [18], and have much in common with the usual translation operator of functions on a group. Convolution of functions can now be defined by

$$
(f * g)(s)=\int_{H} T^{s} f(t) g\left(t^{\vee}\right) d m(t) .
$$

Because of $\mathrm{Hl}$, the convolution is positive, that is $f \geq 0$ and $g \geq 0$ imply $f * g \geq 0$, and so $\|f * g\|_{1} \leq\|f\|_{1}\|g\|_{1}$. Thus the operation makes $L^{1}(\mathrm{dm})$ into a Banach algebra.

The Fourier transform is defined by $f^{\wedge}(\phi)=\int f \phi d m\left(\phi \in H^{\wedge}\right)$; there is a measure $m^{\wedge}$ on $H^{\wedge}$ called Plancherel measure such that

$$
\int_{H}|f|^{2} d m=\int_{H^{\wedge}}\left|f^{\wedge}\right|^{2} d m^{\wedge} \text {. }
$$

The characters define homomorphisms on $M(H)$ and $L^{1}(d m)$ by $h(\mu) \rightarrow$ $\mu^{\wedge}(\phi)$ and $h(f) \rightarrow f^{\wedge}(\phi)$ respectively; in fact, every homomorphism on $L^{1}(\mathrm{dm})$ is of this form.

Many hypergroups arise in connection with product formulas for orthogonal functions on an interval $I$. In particular, suppose $\left\{u_{k}\right\}_{k \in N_{0}} \quad\left(N_{0}=\right.$ $\{0,1,2, \ldots\})$ is a family of continuous functions on $I$, which form a complete 
orthogonal system with respect to a positive measure $m$. A product formula is a relation of the form

$$
\int_{I} u_{k}(r) d \sigma_{s t}(r)=u_{k}(s) u_{k}(t) \quad\left(s, t \in I, k \in N_{0}\right)
$$

where the measure $\sigma_{s t}$ does not depend on $k$. This suggests defining a convolution by identifying $\delta_{s} * \delta_{t}$ with $\sigma_{s t}$, or more generally, if $\mu, \nu \in M(I)$, define $\mu * \nu$ by

$$
\int_{I} f d(\mu * \nu)=\int_{I} \int_{I} \int_{I} f(r) d \sigma_{s t}(r) d \mu(s) d \nu(t) \quad(f \in C(I)) .
$$

If the resulting structure is indeed a hypergroup, then the $u_{k}$ are its Hermitian characters and $m$ is Haar measure for $(I, *)$. Thus the derivation of new product formulas yields new convolution structures and hypergroups; moreover properties of the convolutions so defined may then be used to obtain information about the functions $u_{k}$.

It is often a deep problem to find explicit product formulas for given orthogonal systems or even to provide any general information about the measure in the product formula. In fact, there is a dearth of concrete examples which can be examined to gain insight; the only really well understood examples are the Jacobi polynomials and functions and the associated dual structures $[11,12,13$, 16]. The knowledge we have in those cases is based upon detailed information about the special function systems.

On the other hand, one can expect whole classes of orthogonal systems that admit such structures in the spirit of Connett and Schwartz [6]. One such class is described here as the eigenfunctions of certain Sturm-Liouville problems. The techniques used develop explicit knowledge about the product formulas from the differential operator itself and do not exploit special function knowledge to achieve this end. These techniques will be especially useful in other classical settings (for example in studying product formulas for Mathieu functions) where the eigenfunctions do not have explicit representation (see [5] for details).

We define a class of differential operators on $(0, \pi)$ for $\alpha \geq-1 / 2$ by setting $w^{\alpha}(\theta)=\sin ^{2 \alpha+1} \theta$ and

$$
\begin{aligned}
L_{q, \theta}^{\alpha} & =\frac{1}{w^{\alpha}(\theta)} \frac{d}{d \theta} w^{\alpha}(\theta) \frac{d}{d \theta}-q(\theta) \\
& =\frac{d^{2}}{d \theta^{2}}+\frac{2 \alpha+1}{\tan \theta} \frac{d}{d \theta}-q(\theta)
\end{aligned}
$$

where the potential function $q(\theta)$ is admissible, that is

$$
\begin{gathered}
q(\pi-\theta)=q(\theta) \quad(0<\theta<\pi), \\
\theta q(\theta) \text { is real analytic at } \theta=0, \\
\theta q(\theta) \text { is continuous on }[0, \pi / 2] .
\end{gathered}
$$

The Sturm-Liouville problem is to find $\lambda$ and nontrivial $u_{\lambda}$ which solve the following problem:

$$
\begin{gathered}
L_{q, \theta}^{\alpha} u_{\lambda}(\theta)+\lambda u_{\lambda}(\theta)=0 \quad(0<\theta<\pi), \\
u_{\lambda}^{\prime}(0)=u_{\lambda}^{\prime}(\pi)=0
\end{gathered}
$$


Equation (1.5) is not as restrictive as it seems since any symmetric singular Sturm-Liouville problem with two finite regular singular points having the same exponent difference as the singularities in equation (1.5) can be transformed into that form by changes in the independent and dependent variables.

The boundary conditions (1.6) guarantee that the eigenvalues of $(1.5)-(1.6)$ may be arranged in an unbounded sequence $\lambda_{q, 0}<\lambda_{q, 1}<\cdots<\lambda_{q, k}<\cdots$ (see [6, Lemma 3.3]). Let $u_{q, k}^{\alpha}$ denote the eigenfunction corresponding to $\lambda_{q, k}$ normalized by $u_{q, k}^{\alpha}(0)=1$. The symmetry of the differential operator implies the symmetry of the eigenfunctions in the sense that

$$
u_{q, k}^{\alpha}(\theta)=(-1)^{k} u_{q, k}^{\alpha}(\pi-\theta) \quad\left(0<\theta<\pi, k \in N_{0}\right) .
$$

Moreover, $u_{q, k}^{\alpha}$ has precisely $k$ zeros in $(0, \pi)$. We also have

\subsection{Lemma. $\left\{u_{q, k}^{\alpha}\right\}$ is a complete orthogonal family in $L^{2}\left[w^{\alpha}(\theta) d \theta\right]$.}

Proof. Let $v_{k}(\theta)=\left[w^{\alpha}(\theta)\right]^{1 / 2} u_{q, k}^{\alpha}(\theta)$; then $\lambda=\lambda_{q, 2 k}+\alpha+1 / 2$ and $y=v_{2 k}$ are the eigenvalues and eigenfunctions of the Sturm-Liouville problem

$$
\begin{gathered}
y^{\prime \prime}-Q y+\lambda y=0 \quad(\pi / 2<\theta<\pi), \\
y^{\prime}(\pi / 2)=y(\pi)=0
\end{gathered}
$$

where $Q(\theta)=\left(\alpha^{2}-1 / 4\right) \cot ^{2} \theta+q(\theta)$. Arguments from the proof of [4, Chapter 9, Theorem 3.1 and Problem 1] can be used to establish that $\left\{v_{2 k}\right\}_{k=0}^{\infty}$ is a complete orthogonal family in $L^{2}[(\pi / 2, \pi), d \theta]$. Similarly, one can use the boundary conditions $y(\pi / 2)=y(\pi)=0$ to establish that $\left\{v_{2 k+1}\right\}_{k=0}^{\infty}$ is also a complete orthogonal family in $L^{2}[(\pi / 2, \pi), d \theta]$. Since any function $f \in$ $L^{2}[(0, \pi), d \theta]$ can be decomposed into parts which are even and odd about $\theta=\pi / 2$, it follows that $\left\{v_{k}\right\}_{k=0}^{\infty}$ is complete in $L^{2}[(0, \pi), d \theta]$ and the lemma is established.

The best known example of this class, the ultraspherical equation, is obtained when $q$ is identically zero. This includes Legendre's equation $(\alpha=0)$ and the Chebyshev or cosine equation as a limiting case $(\alpha \rightarrow-1 / 2)$. The corresponding eigenvalues are

$$
\lambda_{k}^{\alpha}=\lambda_{0, k}^{\alpha}=k(k+2 \alpha+1) \quad\left(k \in N_{0}, \alpha \geq-1 / 2\right)
$$

and the eigenfunctions are given in terms of classical functions by

$$
\begin{aligned}
u_{k}^{\alpha}(\theta) & =u_{0, k}^{\alpha}(\theta)=P_{k}^{(\alpha+1 / 2)}(\cos \theta) / P_{k}^{(\alpha+1 / 2)}(1) \\
& =P_{k}^{(\alpha, \alpha)}(\cos \theta) / P_{k}^{(\alpha, \alpha)}(1) \\
& =F\left(-k, k+2 \alpha+1 ; \alpha+1 ; \sin ^{2} \frac{\theta}{2}\right)
\end{aligned}
$$

where $P_{k}^{(\alpha+1 / 2)}$ denotes the ultraspherical polynomial of order $\alpha+1 / 2, P_{k}^{(\alpha, \alpha)}$ is the Jacobi polynomial of order $(\alpha, \alpha)$, and $F$ is Gauss' hypergeometric function. In the limit $(\alpha \rightarrow-1 / 2)$ the eigenfunctions become $u_{k}^{-1 / 2}(\theta)=$ $\cos k \theta$. The orthogonality of the system $\left\{u_{k}^{\alpha}\right\}$ with respect to the weight $w^{\alpha}$ is a direct consequence of the fact that $\left\{u_{k}^{\alpha}\right\}$ is the system of eigenfunctions of the problem (1.5)-(1.6).

Because of the central role played by the ultraspherical case, equation (1.5) will be referred to as a perturbed ultraspherical equation with perturbation $q$. 
Another well-known equation that belongs to this class is the spheroidal wave equation obtained when $q(\theta)=\rho \sin ^{2} \theta \quad(\rho \in R)$. The product formulas for eigenfunctions of the spheroidal wave equation (the spheroidal wave functions) will be treated in detail in a separate paper [5].

Except for trivial cases (e.g. $q$ constant) and the results in [5], the ultraspherical product formula is the only one known explicitly for any $q$ in our class. Before proceeding to the general theory, it will be useful to examine this formula as a model for the general theory.

Let

$$
\begin{aligned}
K^{\alpha}(\xi, \theta, \phi)= & \frac{\Gamma(\alpha+1)}{\Gamma(\alpha+1 / 2) \Gamma(1 / 2)} \\
& \times \frac{([\cos (\theta-\phi)-\cos \xi][\cos \xi-\cos (\theta+\phi)])^{\alpha-1 / 2}}{(\sin \xi \sin \theta \sin \phi)^{2 \alpha}}
\end{aligned}
$$

if $|\theta-\phi|<\xi<\pi-|\theta+\phi-\pi|$ and $K^{\alpha}(\xi, \theta, \phi)=0$ otherwise; then the wellknown formula of Gegenbauer for $\alpha>-1 / 2$ [30, 11.5 or 1, equation (4.10)] may be written

$$
u_{k}^{\alpha}(\theta) u_{k}^{\alpha}(\phi)=\int_{0}^{\pi} u_{k}^{\alpha}(\xi) K^{\alpha}(\xi, \theta, \phi) w^{\alpha}(\xi) d \xi \quad\left(k \in N_{0}, 0<\theta, \phi<\pi\right) .
$$

A product formula in the sense of equation (1.1) is obtained by setting

$$
\begin{gathered}
d \sigma_{\theta \phi}(\xi)=K^{\alpha}(\xi, \theta, \phi) w^{\alpha}(\xi) d \xi \quad(0<\theta, \phi<\pi) \\
\sigma_{0 \theta}=\sigma_{\theta 0}=\delta_{\theta}, \quad \sigma_{\pi \theta}=\sigma_{\theta \pi}=\delta_{\pi-\theta} \quad(0 \leq \theta \leq \pi) .
\end{gathered}
$$

For the cosine case $(\alpha=-1 / 2)$, the product formula is obtained by setting

$$
\sigma_{\theta \phi}=\frac{1}{2}\left[\delta_{|\theta-\phi|}+\delta_{\pi-|\theta+\phi-\pi|}\right] \text {. }
$$

For $\alpha>-1 / 2$, the function $K^{\alpha}(\xi, \theta, \phi)$ is unchanged by permutation of the three variables, it is nonnegative, and setting $k=0$ in equation (1.9) yields

$$
\int_{0}^{\pi} K^{\alpha}(\xi, \theta, \phi) w^{\alpha}(\xi) d \xi=1
$$

With these definitions $([0, \pi], *)$ becomes a commutative hypergroup with identity $\delta_{0}$, involution $\theta^{\vee}=\theta$, characters $\left\{u_{k}^{\alpha}\right\}_{k=0}^{\infty}$, and Haar measure $w^{\alpha}(\xi) d \xi$. Generalized translation of a function $f \in C[0, \pi]$ is given by

$$
\begin{gathered}
T^{\theta} f(\phi)=\int_{0}^{\pi} f(\xi) K^{\alpha}(\xi, \theta, \phi) w^{\alpha}(\xi) d \xi \quad(0<\theta, \phi<\pi), \\
T^{0} f(\phi)=f(\phi) \quad(0 \leq \phi \leq \pi) \\
T^{\pi} f(\phi)=f(\pi-\phi) \quad(0 \leq \phi \leq \pi) .
\end{gathered}
$$

In these cases, the dual structure is also a hypergroup [13].

There are a number of ways of obtaining product formulas, each with a special advantage [1].

1. Formulas analogous to equations (1.8) and (1.9) were first obtained by using knowledge of the special functions involved. These methods are limited in their application. 
2. Many of the special functions occur in a connection with certain topological groups; for instance certain of the ultraspherical polynomials $(\alpha=(n-2) / 2)$ can be used to express the spherical functions on $S O(n)$; thus a convolution for $M[0, \pi]$ is inherited by identifying each $\mu \in M[0, \pi]$ with a bi-invariant measure on $S O(n)$. This ultimately may be used to obtain equation (1.9) by analytic continuation with respect to $\alpha$. The applicability of these methods is limited to those functions which are group-related.

3. Another scheme applicable to eigenfunctions of Sturm-Liouville problems is to use techniques based upon partial differential equations. The advantage is that one can study classes of Sturm-Liouville problems where the potential function is not explicitly given. These ideas go back already to the work of Delsarte [7] and were extended in different directions; e.g., by Levitan [18], Povzner [25], Chébli [3], Braaksma and deSnoo [2], and Trimèche [29], or, in a more abstract setting, by Leblanc [17] and Hutson and Pym [14]. In fact, for any $k \in N_{0}$, let

$$
u(\theta, \phi)=u_{q}(\theta, \phi)=u_{q, k}^{\alpha}(\theta) u_{q, k}^{\alpha}(\phi)
$$

be a product of eigenfunctions of equations (1.5) and (1.6) with arguments belonging to the triangular region

$$
E=\{(\theta, \phi): 0<\phi \leq \theta \leq \pi-\phi<\pi\} .
$$

Then $u$ is the unique solution of the hyperbolic initial value (Cauchy) problem

$$
\begin{gathered}
\left(L_{q, \theta}^{\alpha}-L_{q, \phi}^{\alpha}\right) u(\theta, \phi)=0 \quad((\theta, \phi) \in E), \\
u(\theta, 0)=u_{q, k}^{\alpha}(\theta), \quad u_{\phi}(\theta, 0)=0 \quad(0 \leq \theta \leq \pi) .
\end{gathered}
$$

At this point, it is possible to employ the maximum principle for hyperbolic partial differential equations [31] to prove that the measure $\sigma_{\theta \phi}$ is nonnegative (if appropriate restrictions are placed on $q$ ).

A product formula appears here as a representation of $u$ in terms of initial data, and for this purpose Riemann's integration method [10] is available. This approach, which will be used here, has been successfully employed to obtain new product formulas and to give new proofs of known formulas. (See the papers by one of us $[19,20,21,22,23]$ and the literature there cited.)

The crucial step in the application of Riemann's method is the determination of the Riemann function which is the solution of a characteristic boundary value problem (equation (2.3) below). The kernel function for the product formula is obtained directly from the Riemann function, so information about the Riemann function can be made to yield information about the product formula.

The full product formula for $0<\theta, \phi<\pi$ can be obtained by exploiting the obvious symmetry of $u(\theta, \phi)$ in the two variables as well as the symmetry of the eigenfunctions (equation (1.7)).

The main result of this paper is

1.2. Theorem. Let $\alpha>-1 / 2$ and let $\left\{u_{q, k}^{\alpha}(\theta)\right\}_{k \in N_{0}}$ denote the eigenfunction system related to the Sturm-Liouville problem (1.5)-(1.6) where $q(\theta)$ is supposed to satisfy the conditions (1.2)-(1.4).

(i) For any $\theta, \phi \in(0, \pi)$, the product formula for the eigenfunction system 
is of the form

$$
u_{q, k}^{\alpha}(\theta) u_{q, k}^{\alpha}(\phi)=\int_{0}^{\pi} u_{q, k}^{\alpha}(\xi) D_{q}^{\alpha}(\xi, \theta, \phi) d \xi .
$$

For $(\theta, \phi)$ fixed, $D_{q}^{\alpha}(\xi, \theta, \phi)$ is supported on $(|\theta-\phi|, \pi-|\theta+\phi-\pi|)$ and is continuous there.

(ii) There are constants $A_{q}^{\alpha}$ independent of $\theta$ and $\phi$ such that

$$
\int_{0}^{\pi}\left|D_{q}^{\alpha}(\xi, \theta, \phi)\right| d \xi \leq A_{q}^{\alpha} .
$$

(iii) $D_{q}^{\alpha}(\xi, \theta, \phi) / w^{\alpha}(\xi)$ is symmetric in all three variables.

(iv) If the perturbation function $q(\theta)$ is nonincreasing on $(0, \pi / 2)$, then $D_{q}^{\alpha}(\xi, \theta, \phi)$ is strictly positive on the interval $(|\theta-\phi|, \pi-|\theta+\phi-\pi|)$.

In the particular ultraspherical case $q \equiv 0$, our method yields a new proof of Gegenbauer's product formula, equations (1.8)-(1.9). This is carried out in $\S 2$. In fact, we use the data of the ultraspherical equation to obtain the Riemann function of the corresponding hyperbolic equation (Lemma 2.1), from which the kernel function $D_{0}^{\alpha}(\xi, \theta, \phi)$ can be derived explicitly. A justification of the limiting process is provided in some detail since it gives new insight into the nature of the formula, and it is needed in the argument for the product formula in the general case.

Section 3 is devoted mainly to the proof of Theorem 1.2. Obviously, one can no longer hope to obtain an explicit formula for the Riemann function because the potential function $q$ is only a member of a class. Nevertheless, there is an integral relation connecting the formally defined Riemann function of the perturbed case with the Riemann function of the unperturbed ultraspherical case (equation (3.2)). In fact, this relation will be used to first demonstrate the existence of the perturbed Riemann function, and secondly, as the means of obtaining properties of the perturbed Riemann function from the corresponding properties of the unperturbed function.

Finally in $\S 4$, we turn to harmonic analysis and investigate various convolutions associated with the product formula (1.11). In particular if the kernel $D_{q}^{\alpha}(\xi, \theta, \phi)$ is nonnegative, there is a Jacobi type $(\alpha, \alpha)$ hypergroup (see [6]) with characters $R_{q, k}^{\alpha}(\xi)=u_{q, k}^{\alpha}(\xi) / u_{q, 0}(\xi)$. In this case, it is possible to identify all the complex homomorphisms and all the idempotents of the hypergroup.

\section{A NEW PROOF OF THE PRODUCT FORMULA FOR ULTRASPHERICAL POLYNOMIALS}

Let $\alpha>-1 / 2$ and $k \in N_{0}$. As described in $\S 1$, the product

$$
u(\xi, \eta)=u_{k}^{\alpha}(\xi) u_{k}^{\alpha}(\eta)
$$

is the unique solution of the Cauchy problem (1.10) for the case $q=0$, i.e.,

$$
\begin{gathered}
\left(L_{\xi}^{\alpha}-L_{\eta}^{\alpha}\right) u(\xi, \eta)=0 \quad((\xi, \eta) \in E), \\
u(\xi, 0)=u_{k}^{\alpha}(\xi), \quad u_{\eta}(\xi, 0)=0 \quad(0 \leq \xi \leq \pi),
\end{gathered}
$$

where $L_{\xi}^{\alpha}=L_{0, \xi}^{\alpha}=d^{2} / d \xi^{2}+(2 \alpha+1)(\cot \xi) d / d \xi$. Solving this problem by Riemann's method will yield Gegenbauer's product formula (equations (1.8)(1.9)). 


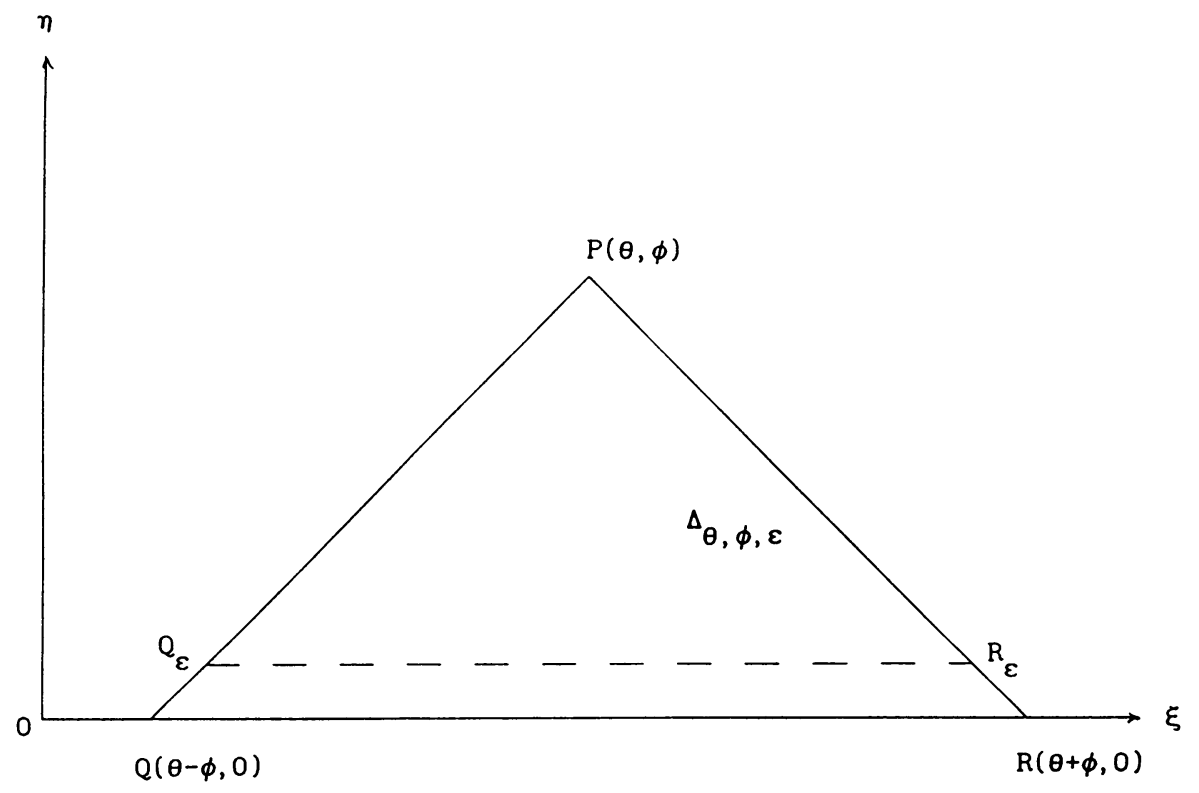

FigURE 1

For any fixed point $(\theta, \phi)$ in the domain $E$, let $\Delta_{\theta \phi}$ denote the characteristic triangle in the $(\xi, \eta)$ plane with vertices $P(\theta, \phi), Q(\theta-\phi, 0)$, and $R(\theta+\phi, 0)$. We also introduce the triangle

$$
\Delta_{\theta \phi \varepsilon}=\left\{(\xi, \eta):(\xi, \eta) \in \Delta_{\theta \phi} \text { and } \eta>\varepsilon\right\} \quad(\varepsilon>0)
$$

with base $\overline{Q_{\varepsilon} R_{\varepsilon}}$ in order to avoid the singularities along the initial line (see Figure 1). The Riemann function associated with the hyperbolic equation in (2.1) is defined as the unique solution

$$
v(\xi, \eta)=v(\xi, \eta ; \theta, \phi)=R^{\alpha}(\xi, \eta ; \theta, \phi)
$$

of the characteristic boundary value problem

$$
\begin{array}{ll}
\left(L_{\xi}^{\alpha}-L_{\eta}^{\alpha}\right)^{*} v(\xi, \eta)=0 \quad\left((\xi, \eta) \in \Delta_{\theta \phi}\right), & \\
v_{\xi}+v_{\eta}-\left(\frac{\alpha+1 / 2}{\tan \xi}+\frac{\alpha+1 / 2}{\tan \eta}\right) v=0 & (\xi-\eta=\theta-\phi), \\
v_{\xi}-v_{\eta}-\left(\frac{\alpha+1 / 2}{\tan \xi}-\frac{\alpha+1 / 2}{\tan \eta}\right) v=0 & (\xi+\eta=\theta+\phi), \\
v(\theta, \phi)=1, &
\end{array}
$$

where the asterisk $(*)$ denotes the adjoint operator. Consequently,

$$
v(\xi, \eta)\left(L_{\xi}^{\alpha}-L_{\eta}^{\alpha}\right) u(\xi, \eta)-u(\xi, \eta)\left(L_{\xi}^{\alpha}-L_{\eta}^{\alpha}\right)^{*} v(\xi, \eta)=0 \quad\left((\xi, \eta) \in \Delta_{\theta \phi}\right) .
$$

By applying Green's theorem to the expression on the left on the triangle $\Delta_{\theta \phi \varepsilon}$, one obtains

$$
\begin{aligned}
u(\theta, \phi)= & \frac{1}{2}\left[\left.(u v)\right|_{Q_{\varepsilon}}+\left.(u v)\right|_{R_{\varepsilon}}\right]+\left.\frac{1}{2} \int_{\theta-\phi+\varepsilon}^{\theta+\phi-\varepsilon}\left[u_{\eta}(\xi, \eta) v(\xi, \eta)\right]\right|_{\eta=\varepsilon} d \xi \\
& +\left.\int_{\theta-\phi}^{\theta+\phi}\left[u(\xi, \eta) D_{\eta}^{\alpha}(\xi, \theta, \phi)\right]\right|_{\eta=\varepsilon} d \xi
\end{aligned}
$$


where

$$
\begin{aligned}
D_{\eta}^{\alpha}(\xi, \theta, \phi) & =-\frac{1}{2}(\sin \eta)^{2 \alpha+1} \frac{\partial}{\partial \eta}\left[(\sin \eta)^{-2 \alpha-1} R^{\alpha}(\xi, \eta ; \theta, \phi)\right] \\
& =\frac{1}{2}\left[\frac{2 \alpha+1}{\tan \eta}-\frac{\partial}{\partial \eta}\right] R^{\alpha}(\xi, \eta ; \theta, \phi),
\end{aligned}
$$

if $\theta-\phi+\eta \leq \xi \leq \theta+\phi-\eta$ and $D_{\eta}^{\alpha}(\xi, \theta, \phi)=0$ otherwise.

In order to obtain equation (1.9), we must first show that the first two terms on the right of (2.4) vanish as $\varepsilon$ tends to $0+$, that for each $\xi, \theta, \phi$

$$
D^{\alpha}(\xi, \theta, \phi)=\lim _{\varepsilon \rightarrow 0+} D_{\varepsilon}^{\alpha}(\xi, \theta, \phi)=K^{\alpha}(\xi, \theta, \phi) w^{\alpha}(\xi),
$$

and that the last term in equation (2.4) converges to the right-hand side of equation (1.9).

The first step in the process is to obtain the Riemann function.

2.1. Lemma. Let $\alpha>-1 / 2$ and $0<\phi \leq \theta \leq \pi-\phi$. The Riemann function associated with equation (2.1) satisfies

$$
R^{\alpha}(\xi, \eta ; \theta, \phi)=\left(\frac{\sin \xi \sin \eta}{\sin \theta \sin \phi}\right)^{\alpha+1 / 2}
$$

on the two characteristics $\xi-\eta=\theta-\phi$ and $\xi+\eta=\theta+\phi$, while for any $(\xi, \eta) \in \Delta_{\theta \phi}$, it is given by

$$
R^{\alpha}(\xi, \eta ; \theta, \phi)=\mathscr{R}^{\alpha}(\xi, \eta ; \theta, \phi) F(1 / 2-|\alpha|, 1 / 2-|\alpha| ; 1 ; \Phi)
$$

where

$$
\begin{gathered}
\mathscr{R}^{\alpha}(\xi, \eta ; \theta, \phi)=\left(\frac{\sin \xi \sin \eta}{\sin \theta \sin \phi}\right)^{\alpha+1 / 2}\left(\frac{\psi}{\omega}\right)^{|\alpha|-1 / 2}, \\
\Phi=\chi / \psi, \\
\chi=[\cos (\theta-\phi)-\cos (\xi-\eta)][\cos (\xi+\eta)-\cos (\theta+\phi)], \\
\psi=[\cos (\theta-\phi)-\cos (\xi+\eta)][\cos (\xi-\eta)-\cos (\theta+\phi)], \\
\omega=4(\sin \xi)(\sin \eta)(\sin \theta)(\sin \phi) .
\end{gathered}
$$

Proof. It will be convenient to make the following substitutions:

$$
\begin{gathered}
X=\cos (\xi+\eta), \quad Y=\cos (\xi-\eta), \\
X_{0}=\cos (\theta+\phi), \quad Y_{0}=\cos (\theta-\phi), \\
v(\xi, \eta ; \theta, \phi)=\left(\frac{\sin \xi \sin \eta}{\sin \theta \sin \phi}\right)^{\alpha+1 / 2} V\left(X, Y ; X_{0}, Y_{0}\right) .
\end{gathered}
$$

Then problem (2.2)-(2.3) reduces to the selfadjoint "normal" form

$$
\begin{gathered}
V_{X Y}+\frac{\alpha^{2}-1 / 4}{(X-Y)^{2}} V=0 \quad\left(X_{0}<X<Y<Y_{0}\right), \\
V\left(X, Y_{0} ; X_{0}, Y_{0}\right)=V\left(X_{0}, Y ; X_{0}, Y_{0}\right)=1 .
\end{gathered}
$$

Thus, equation (2.6) is trivially satisfied on the characteristics. The solution has been given by Riemann [26] in terms of a hypergeometric function:

$$
V\left(X, Y ; X_{0}, Y_{0}\right)=F(1 / 2+\alpha, 1 / 2-\alpha ; 1 ; W)
$$


with the new auxiliary variable

$$
W=W\left(X, Y ; X_{0}, Y_{0}\right)=\frac{\left(X-X_{0}\right)\left(Y-Y_{0}\right)}{(X-Y)\left(X_{0}-Y_{0}\right)} .
$$

The power series on the right-hand side of equation (2.10) converges only for $|W|<1$, but $W$ may take any negative value; however, the function $V$ may still be continued by applying the Pfaff-Kummer transformation:

$$
\begin{aligned}
F(a, b ; c ; z) & =(1-z)^{-a} F\left(a, c-b ; c ; \frac{z}{z-1}\right) \\
& =(1-z)^{-b} F\left(c-a, b ; c ; \frac{z}{z-1}\right)
\end{aligned}
$$

to get

$$
V\left(X, Y ; X_{0}, Y_{0}\right)=(1-W)^{|\alpha|-1 / 2} F\left(\frac{1}{2}-|\alpha|, \frac{1}{2}-|\alpha| ; 1 ; \frac{W}{W-1}\right) .
$$

From this the representation (2.7) of the Riemann function $R^{\alpha}$ follows in view of equation (2.9) and the relations

$$
W=-\frac{\chi}{\omega}, \quad 1-W=\frac{\psi}{\omega}, \quad \frac{W}{W-1}=\Phi .
$$

To obtain the pointwise limit of equation (2.5) and the product formula, the cases $-1 / 2<\alpha \leq 0$ and $\alpha>0$ must be treated separately. We outline the first case; the second will be discussed in less detail.

In view of equation (2.6), $\left.(u v)\right|_{Q_{\varepsilon}} \rightarrow 0$ and $\left.(u v)\right|_{R_{\varepsilon}} \rightarrow 0$ as $\varepsilon \rightarrow 0+$. By application of the identities

$$
\begin{aligned}
& \frac{\partial}{\partial z} F(a, b ; c ; z)=\frac{a \cdot b}{c} F(a+1, b+1 ; c+1 ; z) \\
& F(a, b ; c ; z)=(1-z)^{c-a-b} F(c-a, c-b ; c ; z)
\end{aligned}
$$

we obtain

$$
\begin{array}{r}
D_{\eta}^{\alpha}(\xi, \theta, \phi)=\frac{\alpha+1 / 2}{2} \mathscr{R}^{\alpha}(\xi, \eta ; \theta, \phi) \frac{\partial \psi / \partial \eta}{\psi} F(\alpha+1 / 2, \alpha+1 / 2 ; 1 ; \Phi) \\
-\frac{(\alpha+1 / 2)^{2}}{2} \mathscr{R}^{\alpha}(\xi, \eta ; \theta, \phi) \frac{d \Phi}{d \eta}\left(\frac{\psi}{\omega}\right)^{2 \alpha+1} F(1 / 2-\alpha, 1 / 2-\alpha ; 2 ; \Phi)
\end{array}
$$

so

$$
\begin{aligned}
& u(\theta, \phi)=\frac{1}{2}\left[\left.(u v)\right|_{Q_{\varepsilon}}+\left.(u v)\right|_{R_{\varepsilon}}\right] \\
& \quad+\int_{\theta-\phi}^{\theta+\phi} u_{k}^{\alpha}(\xi)\left[\left(u_{k}^{\alpha}\right)^{\prime}(\varepsilon) T_{1}(\xi, \varepsilon)+\left(u_{k}^{\alpha}\right)(\varepsilon) T_{2}(\xi, \varepsilon)+\left(u_{k}^{\alpha}\right)(\varepsilon) T_{3}(\xi, \varepsilon)\right] d \xi
\end{aligned}
$$

where

$$
\begin{gathered}
T_{1}(\xi, \eta)=\frac{1}{2} R^{\alpha}(\xi, \eta ; \theta, \phi) \\
T_{2}(\xi, \eta)=\frac{\alpha+1 / 2}{2} R^{\alpha}(\xi, \eta ; \theta, \phi) \frac{\partial \psi / \partial \eta}{\psi} \\
T_{3}(\xi, \eta)=-\frac{(\alpha+1 / 2)^{2}}{2} \mathscr{R}^{\alpha}(\xi, \eta ; \theta, \phi) \frac{\partial \Phi}{\partial \eta}\left(\frac{\psi}{\omega}\right)^{2 \alpha+1} F(1 / 2-\alpha, 1 / 2-\alpha ; 2 ; \Phi)
\end{gathered}
$$


if $\theta-\phi+\eta<\xi<\theta+\phi-\eta$ and $T_{1}(\xi, \eta)=T_{2}(\xi, \eta)=T_{3}(\xi, \eta)=0$ elsewhere.

We begin with some preliminary observations. Note that $X_{0} \leq X \leq Y \leq$ $Y_{0}$ where $X, Y, X_{0}$, and $Y_{0}$ are defined in equation (2.8). Since $\psi=$ $\left(Y_{0}-X\right)\left(Y-X_{0}\right)$ it follows that

$$
0 \leq \frac{\partial \psi}{\partial \eta}=\sin (\xi-\eta)\left(Y_{0}-X\right)+\sin (\xi+\eta)\left(Y-X_{0}\right) \leq 2 U_{0}(\sin \xi)
$$

where $U_{0}=Y_{0}-X_{0}=2(\sin \theta)(\sin \phi)$.

Also $\psi=\chi+\omega$; hence

$$
\psi \geq \omega=2 U_{0}(\sin \xi)(\sin \eta) .
$$

It also follows that $\Phi=1-\omega / \psi$; so

$$
\begin{gathered}
\psi \frac{\partial \Phi}{\partial \eta}=-\frac{\partial \omega}{\partial \eta}+\frac{\omega}{\psi} \frac{\partial \psi}{\partial \eta} \\
\left|\psi \frac{\partial \Phi}{\partial \eta}\right| \leq\left(1+\frac{\omega}{\psi}\right) 2 U_{0}(\sin \xi) \leq 4 U_{0}(\sin \xi) .
\end{gathered}
$$

Now we also have

$$
\Phi \rightarrow 1-, \quad \frac{\partial \psi}{\partial \eta} \rightarrow \frac{\omega_{0}}{2}, \quad \frac{\partial \Phi}{\partial \eta} \rightarrow-\frac{\omega_{0}}{\psi_{0}} \quad(\eta \rightarrow 0+)
$$

where

$$
\omega_{0}=4(\sin \xi)(\sin \theta)(\sin \phi), \quad \psi_{0}=[\cos (\theta-\phi)-\cos \xi][\cos \xi-\cos (\theta+\phi)] .
$$

We will also require a Beta integral

$$
\int_{a}^{b}(b-x)^{\gamma-1}(x-a)^{\delta-1} d x=(b-a)^{\gamma+\delta-1} B(\gamma, \delta)
$$

where $B(\gamma, \delta)=\Gamma(\gamma) \Gamma(\delta) / \Gamma(\gamma+\delta)$ is the Beta function, and we introduce the constants

$$
M_{\alpha}= \begin{cases}F(1 / 2-|\alpha|, 1 / 2-|\alpha| ; 1 ; 1)=\Gamma(2|\alpha|) / \Gamma^{2}(1 / 2+|\alpha|) & (\alpha \neq 0), \\ 1 & (\alpha=0) .\end{cases}
$$

We recall that the series for $F(a, b ; c ; z)$ converges absolutely for $|z| \leq 1$ if $\operatorname{Re} c>\operatorname{Re}(a+b)$ and

$$
F(a, b ; c ; 1)=\frac{\Gamma(c) \Gamma(c-a-b)}{\Gamma(c-a) \Gamma(c-b)}
$$

by Gauss' theorem, while in the case $c=a+b$ there are constants $C$ such that

$$
|F(a, b ; c ; z)| \leq 1-C \log (1-z) .
$$

In particular, we have

$$
F(1 / 2-|\alpha|, 1 / 2-|\alpha| ; 1 ; z) \leq \begin{cases}M_{\alpha} & (\alpha \neq 0), \\ 1-\pi^{-1} \log (1-z) & (\alpha=0) .\end{cases}
$$

Thus for $-1 / 2<\alpha \leq 0$,

$$
\mathscr{R}^{\alpha}(\xi, \eta ; \theta, \phi)=\left(\frac{(\sin \xi)(\sin \eta)}{(\sin \theta)(\sin \phi)} \frac{\omega}{\psi}\right)^{\alpha+1 / 2}=\left(\frac{\omega}{U_{0}^{2}} \frac{\omega}{\psi}\right)^{\alpha+1 / 2} ;
$$


hence

Now we have

$$
R^{\alpha}(\xi, \eta ; \theta, \phi) \leq M_{\alpha}\left(\frac{\omega}{U_{0}^{2}}\right)^{\alpha+1 / 2}
$$

$$
\left|T_{1}(\xi, \eta)\right| \leq \frac{1}{2} M_{\alpha}\left(\frac{\omega}{U_{0}^{2}}\right)^{\alpha+1 / 2}
$$

thus

$$
\lim _{\varepsilon \rightarrow 0} \int_{\theta-\phi}^{\theta+\phi}\left|T_{1}(\xi, \varepsilon)\right| d \xi=0
$$

We also have

$$
\begin{aligned}
\left|T_{2}(\xi, \eta)\right| & \leq \frac{\alpha+1 / 2}{U_{0}^{2 \alpha}} M_{\alpha} \frac{\omega^{\alpha+1 / 2}}{\psi} \sin \xi \\
& \leq \frac{\alpha+1 / 2}{U_{0}^{2 \alpha}} M_{\alpha} \psi_{0}^{\alpha-1 / 2} \sin \xi .
\end{aligned}
$$

The first inequality shows that for each $\xi, \lim _{\varepsilon \rightarrow 0} T_{2}(\xi, \varepsilon)=0$. The second bound which is independent of $\eta$ yields

$$
\begin{aligned}
\int_{\theta-\phi}^{\theta+\phi} & \left|T_{2}(\xi, \eta)\right| d \xi \leq(\alpha+1 / 2) M_{\alpha} U_{0}^{-2 \alpha} \int_{\theta-\phi}^{\theta+\phi} \psi_{0}^{\alpha-1 / 2} \sin \xi d \xi \\
& =(\alpha+1 / 2) M_{\alpha} U_{0}^{-2 \alpha} \int_{X_{0}}^{Y_{0}}\left[\left(Y_{0}-t\right)\left(t-X_{0}\right)\right]^{\alpha-1 / 2} d t \\
& =(\alpha+1 / 2) M_{\alpha} B(\alpha+1 / 2, \alpha+1 / 2) .
\end{aligned}
$$

Up to a constant factor $\left|T_{3}(\xi, \eta)\right|$ has the same upper bound as $\left|T_{2}(\xi, \eta)\right|$. In fact,

$$
\begin{aligned}
\left|T_{3}(\xi, \eta)\right| & \leq \frac{(\alpha+1 / 2)^{2}}{2}\left(\frac{\omega}{U_{0}^{2}} \frac{\omega}{\psi}\right)^{\alpha+1 / 2}\left|\frac{\partial \Phi}{\partial \eta}\right|\left(\frac{\psi}{\omega}\right)^{2 \alpha+1} F(1 / 2-\alpha, 1 / 2-\alpha ; 2 ; \Phi) \\
& \leq \frac{(\alpha+1 / 2)^{2}}{2} F(1 / 2-\alpha, 1 / 2-\alpha ; 2 ; 1) U_{0}^{-2 \alpha-1}\left|\frac{\partial \Phi}{\partial \eta}\right| \psi^{\alpha+1 / 2} \\
& \leq \frac{(\alpha+1 / 2)^{2}}{2} \frac{\Gamma(2 \alpha+1)}{\Gamma^{2}(\alpha+3 / 2)} U_{0}^{-2 \alpha-1} 4 U_{0}(\sin \xi) \psi_{0}^{\alpha-1 / 2} \\
& \leq 2 \frac{\Gamma(2 \alpha+1)}{\Gamma^{2}(\alpha+1 / 2)} U_{0}^{-2 \alpha} \psi_{0}^{\alpha-1 / 2} \sin \xi
\end{aligned}
$$

whence

$$
\int_{\theta-\phi}^{\theta+\phi}\left|T_{3}(\xi, \eta)\right| d \xi \leq 2
$$

Together these yield

$$
\begin{aligned}
\left|D_{\eta}^{\alpha}(\xi, \theta, \phi)\right| & \leq \mathscr{D}^{\alpha}(\xi, \theta, \phi) \\
& =\left((\alpha+1 / 2) M_{\alpha}+\frac{2 \Gamma(2 \alpha+1)}{\Gamma^{2}(\alpha+1 / 2)}\right) U_{0}^{-2 \alpha} \psi_{0}^{\alpha-1 / 2} \sin \xi
\end{aligned}
$$

and

$$
\int_{\theta-\phi}^{\theta+\phi} \mathscr{D}^{\alpha}(\xi, \theta, \phi) d \xi \leq \mathscr{B}_{\alpha}=(\alpha+1 / 2) M_{\alpha} B(\alpha+1 / 2, \alpha+1 / 2)+2
$$


(Note that the bound $\mathscr{B}_{\alpha}$ is independent of $\eta, \theta$, and $\phi$; this will be used in §3.)

For $-1 / 2<\alpha \leq 0$, identity (2.5) now follows by

$$
\begin{aligned}
D^{\alpha}(\xi, \theta, \phi) & =\lim _{\varepsilon \rightarrow 0} T_{3}(\xi, \varepsilon) \\
& =\frac{(\alpha+1 / 2)^{2}}{2} \omega_{0} \psi_{0}^{\alpha-1 / 2} U_{0}^{-2 \alpha-1} F(1 / 2-\alpha, 1 / 2-\alpha ; 2 ; 1) \\
& =\frac{\Gamma(2 \alpha+1)}{2^{2 \alpha} \Gamma^{2}(\alpha+1 / 2)} \frac{\psi_{0}^{\alpha-1 / 2} w^{\alpha}(\xi)}{\sin \xi \sin \theta \sin \phi)^{2 \alpha}} \\
& =K^{\alpha}(\xi, \theta, \phi) w^{\alpha}(\xi),
\end{aligned}
$$

where in the last step, Legendre's duplication formula for the gamma function has been used. Equation (1.9) follows by Lebesgue's Dominated Convergence Theorem.

For $\alpha>0$, begin by applying only equation (2.11) to the second expression for $D_{\eta}^{\alpha}(\xi, \theta, \phi)$ to obtain

$$
\begin{aligned}
D_{\eta}^{\alpha}(\xi, \theta, \phi)= & \alpha(\cot \eta) \mathscr{R}^{\alpha}(\xi, \eta ; \theta, \phi) F(1 / 2-\alpha, 1 / 2-\alpha ; 1 ; \Phi) \\
& -\frac{1}{2}(\alpha-1 / 2) \mathscr{R}^{\alpha}(\xi, \eta ; \theta, \phi) \frac{\partial \psi / \partial \eta}{\psi} F(1 / 2-\alpha, 1 / 2-\alpha ; 1 ; \Phi) \\
& -\frac{1}{2}(1 / 2-\alpha)^{2} \mathscr{R}^{\alpha}(\xi, \eta ; \theta, \phi) \frac{\partial \Phi}{\partial \eta} F(3 / 2-\alpha, 3 / 2-\alpha ; 2 ; \Phi) .
\end{aligned}
$$

The analysis here resembles that for $\alpha \leq 0$; the only item of special note is that $F(3 / 2-\alpha, 3 / 2-\alpha ; 2 ; \Phi)$ is bounded for $\alpha>1 / 2$, while if $0<\alpha \leq 1 / 2$, the transformation (2.12) is required. The result is that all the terms except the first in the expression for $D_{\eta}^{\alpha}(\xi, \theta, \phi)$ tend to zero as $\eta \rightarrow 0+$, and the first one yields the desired limit.

\section{Proof of Theorem 1.2}

By symmetry, it will suffice to consider only $(\theta, \phi) \in E$. Let $R_{q}^{\alpha}(s, t ; \theta, \phi)$ be the Riemann function for the hyperbolic equation in (1.10), that is $v(s, t)=$ $R_{q}^{\alpha}(s, t ; \theta, \phi)$ satisfies

$$
\begin{aligned}
\left(L_{s}^{\alpha}-L_{t}^{\alpha}\right)^{*} v(s, t)-Q(s, t) v(s, t)=0 \quad((s, t) & \left.\in \Delta_{\theta \phi}\right), \\
v_{s}(s, t)+v_{t}(s, t)-\left(\frac{\alpha+1 / 2}{\tan s}+\frac{\alpha+1 / 2}{\tan t}\right) v(s, t) & =0 \\
(s-t=\theta-\phi), & \\
v_{s}(s, t)-v_{t}(s, t)-\left(\frac{\alpha+1 / 2}{\tan s}-\frac{\alpha+1 / 2}{\tan t}\right) v(s, t) & =0 \\
v(\theta, \phi) & =1,
\end{aligned}
$$

where $Q(s, t)=q(s)-q(t)$. In view of the reciprocity law for Riemann functions (cf. [10, equation (4.80)]), one knows that as a function of $(s, t)$, the Riemann function $R^{\alpha}(\xi, \eta ; s, t)$ is the Riemann function for the adjoint equation, 


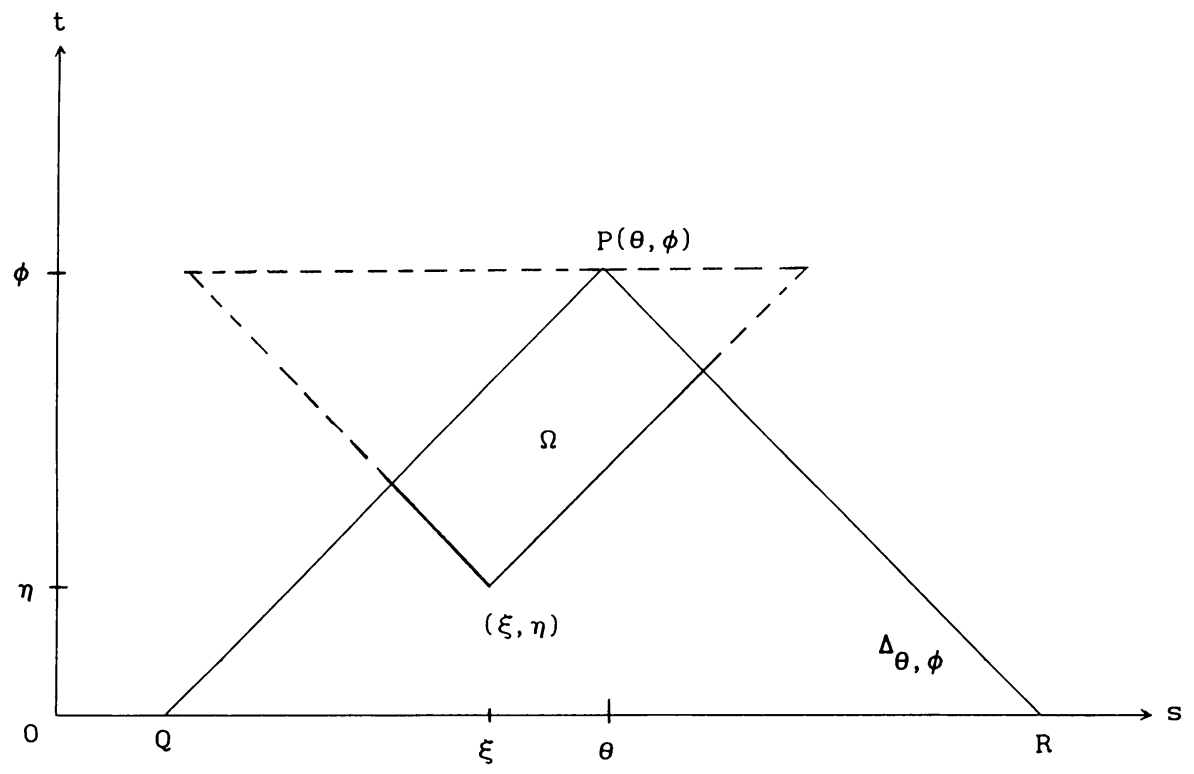

FIGURE 2

i.e., $u(s, t)=R^{\alpha}(\xi, \eta ; s, t)$ satisfies

$$
\begin{gathered}
\left(L_{s}^{\alpha}-L_{t}^{\alpha}\right) u(s, t)=0 \quad\left((s, t) \text { such that }(\xi, \eta) \in \Delta_{s t}\right), \\
u_{s}(s, t)+u_{t}(s, t)+\left(\frac{\alpha+1 / 2}{\tan s}+\frac{\alpha+1 / 2}{\tan t}\right) u(s, t)=0 \quad(s-t=\xi-\eta), \\
u_{s}(s, t)-u_{t}(s, t)+\left(\frac{\alpha+1 / 2}{\tan s}-\frac{\alpha+1 / 2}{\tan t}\right) u(s, t)=0 \quad(s+t=\xi+\eta), \\
u(\xi, \eta)=1 .
\end{gathered}
$$

2)

If $(\xi, \eta) \in \Delta_{\theta \phi}$, then both equations hold in the tilted rectangle (see Figure

$$
\begin{aligned}
\Omega & =\Omega(\xi, \eta ; \theta, \phi)=\left\{(s, t):(s, t) \in \Delta_{\theta \phi} \text { and }(\xi, \eta) \in \Delta_{s t}\right\} \\
& =\{(s, t): \theta-\phi<s-t<\xi-\eta, \xi+\eta<s+t<\theta+\phi\} .
\end{aligned}
$$

By applying Green's theorem on $\Omega$ to the quantity

$$
\begin{aligned}
B(s, t) & =v(s, t)\left(L_{s}^{\alpha}-L_{t}^{\alpha}\right) u(s, t)-u(s, t)\left(L_{s}^{\alpha}-L_{t}^{\alpha}\right)^{*} v(s, t) \\
& =-u(s, t) Q(s, t) v(s, t) \\
& =-R^{\alpha}(\xi, \eta ; s, t) Q(s, t) R_{q}^{\alpha}(s, t ; \theta, \phi),
\end{aligned}
$$

one obtains an integral equation linking $R^{\alpha}$ and $R_{q}^{\alpha}$, namely

$$
\begin{aligned}
& R_{q}^{\alpha}(\xi, \eta ; \theta, \phi)=R^{\alpha}(\xi, \eta ; \theta, \phi) \\
& \quad-\frac{1}{2} \iint_{\Omega} R^{\alpha}(\xi, \eta ; s, t) Q(s, t) R_{q}^{\alpha}(s, t ; \theta, \phi) d s d t
\end{aligned}
$$

which is valid for any $(\xi, \eta) \in \Delta_{\theta \phi}$ (cf., e.g., [10]). We will use successive approximation to show that equation (3.2) in the unknown function $R_{q}^{\alpha}$ has a unique solution. This will require two technical lemmas (cf. also [2 and 21]). First observe that, by equations (2.7), (2.14), and (2.15)

$$
0<R^{\alpha}(\xi, \eta ; \theta, \phi) \leq M_{\alpha} h_{\alpha}(\eta) \mathscr{R}^{\alpha}(\xi, \eta ; \theta, \phi)
$$


where $M_{\alpha}$ are defined by equation $(2.14), h_{0}(\eta)=1-2 \pi^{-1} \log (\sin \eta)$ and, $h_{\alpha}(\eta)=1$ if $\alpha \neq 0$.

It will be more convenient to normalize (3.2) by dividing by $R^{\alpha}(\xi, \eta ; \theta, \phi)$ to obtain

$$
r(\xi, \eta)=1+J[r](\xi, \eta) \quad\left(\xi, \eta \in \Delta_{\theta \phi}\right)
$$

where

$$
r(\xi, \eta)=\frac{R_{q}^{\alpha}(\xi, \eta ; \theta, \phi)}{R^{\alpha}(\xi, \eta ; \theta, \phi)}
$$

and

$$
\begin{aligned}
J[r](\xi, \eta)= & -\frac{1}{2 R^{\alpha}(\xi, \eta ; \theta, \phi)} \\
& \cdot \iint_{\Omega(\xi, \eta ; \theta, \phi)} R^{\alpha}(\xi, \eta ; s, t) Q(s, t) r(s, t) R^{\alpha}(s, t ; \theta, \phi) d s d t .
\end{aligned}
$$

3.1. Lemma. Let $\alpha>-1 / 2$. For $P(t)$ continuous and nonnegative in $(0, \pi / 2)$ define

$$
\begin{aligned}
I[P] & =I[P](\xi, \eta) \\
& =\frac{1}{2 R^{\alpha}(\xi, \eta ; \theta, \phi)} \iint_{\Omega(\xi, \eta ; \theta, \phi)} R^{\alpha}(\xi, \eta ; s, t) P(t) R^{\alpha}(s, t ; \theta, \phi) d s d t
\end{aligned}
$$

Then there is a constant $K_{\alpha}$ such that

$$
I[P] \leq K_{\alpha} \int_{\eta}^{\phi} t h_{\alpha}(t) P(t) d t .
$$

Proof. We begin by showing

$$
\frac{R^{\alpha}(\xi, \eta ; s, t)}{R^{\alpha}(\xi, \eta ; \theta, \phi)} \leq \frac{\mathscr{R}^{\alpha}(\xi, \eta ; s, t)}{\mathscr{R}^{\alpha}(\xi, \eta ; \theta, \phi)} .
$$

To see this, let $X, Y, X_{0}$, and $Y_{0}$ be as in equation (2.8) and let $S=\cos (s+t)$ and $T=\cos (s-t)$; then

$$
\begin{aligned}
\rho & =\frac{1-\Phi(\xi, \eta ; \theta, \phi)}{1-\Phi(\xi, \eta ; s, t)}=\frac{\omega(\xi, \eta ; \theta, \phi)}{\psi(\xi, \eta ; \theta, \phi)} \frac{\psi(\xi, \eta ; s, t)}{\omega(\xi, \eta ; s, t)} \\
& =\frac{\left(Y_{0}-X_{0}\right)(T-X)(Y-S)}{(T-S)\left(Y_{0}-X\right)\left(Y-X_{0}\right)} .
\end{aligned}
$$

Now use successively,

$$
\begin{gathered}
\frac{Y_{0}-X}{Y_{0}-S}-\frac{T-X}{T-S}=\frac{(X-S)\left(Y_{0}-T\right)}{\left(Y_{0}-S\right)(T-S)}>0, \\
\frac{Y-X_{0}}{Y_{0}-X_{0}}-\frac{Y-S}{Y_{0}-S}=\frac{\left(S-X_{0}\right)\left(Y_{0}-Y\right)}{\left(Y_{0}-X_{0}\right)\left(Y_{0}-S\right)}>0,
\end{gathered}
$$

to obtain $0<\rho \leq 1$. Thus $0<\Phi(\xi, \eta ; s, t) \leq \Phi(\xi, \eta ; \theta, \phi) \leq 1$ so that

$$
\begin{aligned}
& F(1 / 2-|\alpha|, 1 / 2-|\alpha| ; 1 ; \Phi(\xi, \eta ; s, t)) \\
& \quad \leq F(1 / 2-|\alpha|, 1 / 2-|\alpha| ; 1 ; \Phi(\xi, \eta ; \theta, \phi))
\end{aligned}
$$


since $F(1 / 2-|\alpha|, 1 / 2-|\alpha| ; 1 ; x)$ is an increasing function on $0<x<1$ and the promised inequality is established; so it follows that

$$
I[P] \leq \frac{1}{2} M_{\alpha} \iint_{\Omega}[A(s, t)]^{|\alpha|-1 / 2} h_{\alpha}(t) P(t) d s d t
$$

where

$$
A(s, t)=\frac{\psi(\xi, \eta ; s, t) \psi(s, t ; \theta, \phi)}{\psi(\xi, \eta ; \theta, \phi) 4 \sin ^{2} s \sin ^{2} t}
$$

For $\alpha \geq 1 / 2$, define $X, Y, X_{0}$, and $Y_{0}$ as in equation (2.8) and let $S=$ $\cos (s+t)$ and $T=\cos (s-t)$; then

$$
A(s, t)=\left[1-\frac{(X-S)\left(Y_{0}-T\right)}{\left(Y_{0}-X\right)(T-S)}\right]\left[1-\frac{\left(S-X_{0}\right)(T-Y)}{\left(Y-X_{0}\right)(T-S)}\right] .
$$

Because $-1<X_{0}<S<X<Y<T<Y_{0}<1$, the quotients all lie in $(0,1)$, so $A(s, t) \leq 1$, hence,

$$
I[P] \leq \frac{1}{2} M_{\alpha} \iint_{\Omega} h_{\alpha}(t) P(t) d s d t .
$$

Now, since $P(t) \geq 0$, the integral is only increased if $\Omega$ is replaced by the larger triangular region with vertices $(\xi, \eta),(\xi+\eta-\phi, \phi)$, and $(\xi-\eta+\phi, \phi)$ (see Figure 2) to obtain the lemma with $K_{\alpha}=M_{\alpha}$.

When $|\alpha|<1 / 2$, we require a lower bound for $A(s, t)$. Using the identity

$$
\cos a-\cos b=2 \sin \left(\frac{b+a}{2}\right) \sin \left(\frac{b-a}{2}\right)
$$

we obtain with a little rearranging

$$
\begin{aligned}
A(s, t)= & \frac{\sin \frac{s+t-(\theta-\phi)}{2} \sin \frac{\theta+\phi-(s-t)}{2}}{\sin \frac{\xi+\eta-(\theta-\phi)}{2} \sin \frac{\theta+\phi-(\xi-\eta)}{2}} \times \frac{\sin \frac{\xi+\eta+s-t}{2} \sin \frac{s+t+\theta-\phi}{2}}{\sin \frac{\xi+\eta+\theta-\phi}{2} \sin s} \\
& \times \frac{\sin \frac{s+t+\xi-\eta}{2} \sin \frac{\theta+\phi+s-t}{2}}{\sin \frac{\theta+\phi+\xi-\eta}{2} \sin s} \times \frac{\sin \frac{s+t-(\xi-\eta)}{2} \sin \frac{\xi+\eta-(s-t)}{2}}{\sin ^{2} t} \\
= & f_{1} \times f_{2} \times f_{3} \times f_{4} .
\end{aligned}
$$

Now,

$$
0 \leq \frac{\theta-\phi}{2}<\frac{s-t}{2}<\frac{\xi-\eta}{2}<\frac{\xi+\eta}{2}<\frac{s+t}{2}<\frac{\theta+\phi}{2} \leq \frac{\pi}{2} \quad((s, t) \in \Omega),
$$

hence, $f_{1}>1$. To estimate $f_{2}$ and $f_{3}$ from below, observe that for any $0 \leq a \leq b \leq c \leq d \leq \pi / 2$,

$$
\frac{\sin (a+d) \sin (b+c)}{\sin (a+c) \sin (b+d)}=1+\frac{\sin (b-a) \sin (d-c)}{\sin (a+c) \sin (b+d)} \geq 1 \text {. }
$$

Choosing

$$
(a, b, c, d)=\left(\frac{\theta-\phi}{2}, \frac{s-t}{2}, \frac{\xi+\eta}{2}, \frac{s+t}{2}\right)
$$

and

$$
\left(\frac{s-t}{2}, \frac{\xi-\eta}{2}, \frac{s+t}{2}, \frac{\theta+\phi}{2}\right)
$$


this yields $f_{2} \geq 1$ and $f_{3} \geq 1$ respectively. Finally, we use $2 x / \pi \leq \sin x \leq x$ $(0 \leq x \leq \pi / 2)$ in $f_{4}$ to obtain

$$
\begin{aligned}
A(s, t)>f_{4} & \geq(\pi t)^{-2}\left[(t+\eta)^{2}-(s-\xi)^{2}\right] \\
& \geq(\pi t)^{-2}\left[(t-\eta)^{2}-(s-\xi)^{2}\right] \geq 0 .
\end{aligned}
$$

Thus, proceeding as in the case $\alpha \geq 1 / 2$, we have

$$
I[P] \leq \frac{1}{2} M_{\alpha} \pi^{1-2|\alpha|} \int_{\eta}^{\phi} \int_{\xi+\eta-t}^{\xi-\eta+t}\left[(t-\eta)^{2}-(s-\xi)^{2}\right]^{|\alpha|-1 / 2} d s t^{1-2|\alpha|} h_{\alpha}(t) P(t) d t .
$$

The inner integral can be evaluated exactly as a Beta function to finally yield the lemma with

$$
K_{\alpha}=\frac{1}{2} M_{\alpha} \pi^{3 / 2-2|\alpha|} \frac{\Gamma(|\alpha|+1 / 2)}{\Gamma(2|\alpha|+1)} .
$$

Let $J^{0}[f]=f$, and $J^{k}[f]=J\left[J^{k-1}[f]\right](k \in N),\|g\|_{\Omega}=\sup |g(s, t)|$ $((s, t) \in \Omega)$, and

$$
H_{\alpha}(\eta)=H_{\alpha}(\eta, \phi)=\int_{\eta}^{\phi} t h_{\alpha}(t) Q(t) d t
$$

where $h_{\alpha}$ is as in equation (3.3) and $Q(t)$ is an upper bound for $|Q(s, t)|(t \leq$ $s \leq \pi-t)$, so by $(1.4) H_{\alpha}(\eta, \phi)$ is uniformly bounded for $0<\eta<\phi<\pi / 2$.

3.2. Lemma. Let $\alpha>-1 / 2$ and $(\xi, \eta) \in \Delta_{\theta \phi}$. For any $f$ uniformly bounded on $\Omega=\Omega(\xi, \eta ; \theta, \phi)$,

$$
\left\|J^{k}[f]\right\|_{\Omega} \leq\|f\|_{\Omega} \frac{\left(K_{\alpha} H_{\alpha}(\eta)\right)^{k}}{k !} \quad\left(k \in N_{0}\right)
$$

where $K_{\alpha}$ is as in Lemma 3.1.

Proof. We prove the lemma by induction; it is trivially true for $k=0$. We now assume equation (3.5) is true for a particular value of $k$ and apply it with $(s, t)$ in place of $(\xi, \eta)$ to obtain

$$
\begin{aligned}
\left|J^{k+1}[f](s, t)\right| & =\frac{1}{2 R^{\alpha}(s, t ; \theta, \phi)} \mid \iint_{\Omega(s, t ; \theta, \phi)} R^{\alpha}(s, t ; \sigma, \tau) Q(\sigma, \tau) J^{k}[f](\sigma, \tau) \\
& \leq \frac{\|f\|_{\Omega(s, t ; \theta, \phi)}}{2 R^{\alpha}(s, t ; \theta, \phi)} \iint_{\Omega(s, t ; \theta, \phi)} R^{\alpha}(\sigma, t ; \sigma, \tau) Q(\tau) \frac{\left(K_{\alpha} H_{\alpha}(\tau)\right)^{k}}{k !} \\
& =\|f\|_{\Omega(s, t ; \theta, \phi)} I\left[Q(\tau) \frac{\left(K_{\alpha} H_{\alpha}(\tau)\right)^{k}}{k !}\right](s, t) \\
& \leq\|f\|_{\Omega(s, t ; \theta, \phi)} \frac{K_{\alpha}^{k+1}}{k !} \int_{\tau}^{\phi} \tau h_{\alpha}(\tau) Q(\tau)\left[\int_{\tau}^{\alpha}(\sigma, \tau ; \theta, \phi) d \sigma d \tau\right. \\
& \left.\leq\|f\|_{\Omega(s, t ; \theta, \phi)} \frac{\left[K_{\alpha} H_{\alpha}(t)\right]^{k+1}}{(k+1) !} Q(r) d r\right]^{k} d \tau
\end{aligned}
$$


by Lemma 3.1 and the Fundamental Theorem of Calculus. This proves a pointwise inequality. To obtain the norm inequality, observe that if $(s, t) \in$ $\Omega(\xi, \eta ; \theta, \phi)$ then $\Omega(s, t ; \theta, \phi) \subseteq \Omega(\xi, \eta ; \theta, \phi)$. Thus to estimate the last expression above replace $(s, t)$ by $(\xi, \eta)$ and take the supremum on the lefthand side.

We now obtain a bound for the perturbed Riemann function.

3.3. Lemma. (i) If $q$ is an admissible potential function ((1.2)-(1.4)) then $R_{q}^{\alpha}$ is the unique solution of equation (3.2), it is continuous and

$$
\left|R_{q}^{\alpha}(\xi, \eta ; \theta, \phi)\right| \leq R^{\alpha}(\xi, \eta ; \theta, \phi) \exp \left(K_{\alpha} H_{\alpha}(\eta)\right)
$$

where $K_{\alpha}$ and $H_{\alpha}$ are as in Lemma 3.2.

(ii) If in addition to being admissible, $q$ is monotone decreasing in $(0, \pi / 2)$, then $R_{q}^{\alpha}(\xi, \eta ; \theta, \phi) \geq 0$.

Proof. We will show that (3.4) has a unique solution $r(\xi, \eta)=r(\xi, \eta ; \theta, \phi)$ which is continuous in $(\xi, \eta)$ and $(\theta, \phi)$, and that

$$
\|r\|_{\Omega} \leq \exp \left[K_{\alpha} H_{\alpha}(\eta)\right] .
$$

Thus part (i) becomes a simple conclusion from the definition of $r$.

We now return to equation (3.4); define $r_{0}=1$ and

$$
r_{k+1}=1+J\left[r_{k}\right] \quad\left(k \in N_{0}\right) .
$$

By Lemma 3.2,

$$
\left\|r_{k}-r_{k-1}\right\|_{\Omega}=\left\|J^{k-1}\left[r_{1}-1\right]\right\|_{\Omega}=\left\|J^{k}[1]\right\|_{\Omega} \leq \frac{\left[K_{\alpha} H_{\alpha}(\eta)\right]^{k}}{k !} .
$$

So $\sum_{j=1}^{\infty}\left(r_{j}-r_{j-1}\right)$ converges uniformly on $\Omega$. Let

$$
r=\lim _{k \rightarrow \infty} r_{k}=\lim _{k \rightarrow \infty} \sum_{j=1}^{k}\left(r_{j}-r_{j-1}\right)+1 ;
$$

then if $k$ is increased without bound in equation (3.6), we obtain equation (3.4), and existence is established.

To prove uniqueness, assume $\tilde{r}=1+J[\tilde{r}]$; then $r-\tilde{r}=J[r-\tilde{r}]$, so $r-\tilde{r}=$ $J^{k}[r-\tilde{r}] \quad(k \in N)$; Lemma 3.2 then yields

$$
\|r-\tilde{r}\|_{\Omega} \leq\|r-\tilde{r}\|_{\Omega} \frac{\left[K_{\alpha} H_{\alpha}(\eta)\right]^{k}}{k !} \quad(k \in N)
$$

which is possible only if $r=\tilde{r}$. Finally, equation (3.8) followed by equation (3.7) yields

$$
\|r\|_{\Omega} \leq \sum_{k=1}^{\infty}\left\|r_{k}-r_{k-1}\right\|_{\Omega}+1 \leq \exp \left[K_{\alpha} H_{\alpha}(\eta)\right] .
$$

For part (ii), we already know $R^{\alpha}$ is nonnegative, and the additional condition entails $Q(s, t) \leq 0$ so $J[f]$ is nonnegative whenever $f$ is. It follows that $r_{k}$ are all nonnegative, hence so must be $r$. 
Proof of Theorem 1.2. As in the ultraspherical case (equation (2.4)) we obtain for $u(\theta, \phi)=u_{q, k}^{\alpha}(\theta) u_{q, k}^{\alpha}(\phi), v(s, t)=R_{q}^{\alpha}(s, t ; \theta, \phi)$,

$$
\begin{aligned}
u(\theta, \phi)= & \frac{1}{2}\left[\left.(u v)\right|_{Q_{\varepsilon}}+\left.(u v)\right|_{R_{\varepsilon}}\right] \\
& +\left.\frac{1}{2} \int_{\theta-\phi+\varepsilon}^{\theta+\phi-\varepsilon}\left[u_{\eta}(\xi, \eta) v(\xi, \eta)\right]\right|_{\eta=\varepsilon} d \xi \\
& +\left.\int_{\theta-\phi}^{\theta+\phi}\left[u(\xi, \eta) D_{q, \eta}^{\alpha}(\xi, \theta, \phi)\right]\right|_{\eta=\varepsilon} d \xi
\end{aligned}
$$

where now

$$
D_{q, \eta}^{\alpha}(\xi, \theta, \phi)=-\frac{1}{2}(\sin \eta)^{2 \alpha+1} \frac{\partial}{\partial \eta}\left[(\sin \eta)^{-2 \alpha-1} R_{q}^{\alpha}(\xi, \eta ; \theta, \phi)\right]
$$

if $\theta-\phi+\eta \leq \xi \leq \theta+\phi-\eta$ and $D_{q, \eta}^{\alpha}(\xi, \theta, \phi)=0$ elsewhere.

Now by the same argument as in Lemma 2.1, equation (2.6) with $R_{q}^{\alpha}$ in place of $R^{\alpha}$ holds on the two characteristics $\xi-\eta=\theta-\phi$ and $\xi+\eta=\theta+\phi$. Thus the first term on the right side of (3.9) vanishes as $\varepsilon \rightarrow 0+$. Lemma 3.3 can be used to show that the second term vanishes $\varepsilon \rightarrow 0+$ because of the analogous result in the ultraspherical case. From equation (3.2),

$$
D_{q, \eta}^{\alpha}(\xi, \theta, \phi)=D_{\eta}^{\alpha}(\xi, \theta, \phi)+\frac{1}{4}(\sin \eta)^{2 \alpha+1} \frac{\partial}{\partial \eta} \iint_{\Omega} g(\xi, \eta ; s, t) d s d t
$$

where

$$
g(\xi, \eta ; s, t)=(\sin \eta)^{-2 \alpha-1} R^{\alpha}(\xi, \eta ; s, t) Q(s, t) R_{q}^{\alpha}(s, t ; \theta, \phi) .
$$

We introduce the change of variables $\sigma=s+t, \tau=s-t$ and obtain for the second term in (3.11) the sum

$$
\begin{aligned}
-\int_{\theta-\phi}^{\xi-\eta} \frac{1}{8}(\sin \eta)^{2 \alpha+1} g\left(\xi, \eta ; \frac{\xi+\eta+\tau}{2}, \frac{\xi+\eta-\tau}{2}\right) d \tau \\
-\int_{\xi+\eta}^{\theta+\phi} \frac{1}{8}(\sin \eta)^{2 \alpha+1} g\left(\xi, \eta ; \frac{\sigma+\xi-\eta}{2}, \frac{\sigma-\xi+\eta}{2}\right) d \sigma \\
+\int_{\xi+\eta}^{\theta+\phi} \int_{\theta-\phi}^{\xi-\eta} \frac{1}{8}(\sin \eta)^{2 \alpha+1} \frac{\partial g}{\partial \eta}\left(\xi, \eta ; \frac{\sigma+\tau}{2}, \frac{\sigma-\tau}{2}\right) d \tau d \sigma \\
=I_{1}(\xi, \eta)+I_{2}(\xi, \eta)+I_{3}(\xi, \eta) .
\end{aligned}
$$

Equation (1.11) with

$$
\begin{aligned}
& D_{q}^{\alpha}(\xi, \theta, \phi)=\lim _{\eta \rightarrow 0+} D_{q, \eta}^{\alpha}(\xi, \theta, \phi)=D^{\alpha}(\xi, \theta, \phi) \\
& \quad-\frac{1}{2} \iint_{\Omega(\xi, 0 ; \theta, \phi)} D^{\alpha}(\xi, s, t) Q(s, t) R_{q}^{\alpha}(s, t ; \theta, \phi) d s d t
\end{aligned}
$$

will be obtained by the Lebesgue Dominated Convergence Theorem.

This argument has the same sources of complexity as that for the ultraspherical product formula, so we will limit our attention to the more difficult case of $-1 / 2<\alpha \leq 0$. 
To estimate $\int_{\theta-\phi}^{\theta+\phi}\left|I_{1}(\xi, \eta)\right| d \xi$ observe that

$$
|Q(s, t)| \leq C\left(\frac{1}{\sin s}+\frac{1}{\sin t}\right)
$$

so

$$
(\sin \eta)^{2 \alpha+1}|g(\xi, \eta ; s, t)| \leq M_{\alpha}^{\prime} \frac{\sin s+\sin t}{(\sin s)(\sin t)}\left(\frac{2(\sin \xi)(\sin \eta)}{U_{0}}\right)^{\alpha+1 / 2}
$$

where $M_{\alpha}^{\prime}=C M_{\alpha}^{2} \exp \left[K_{\alpha} H_{\alpha}(\eta)\right], s=(\xi+\eta+\tau) / 2$, and $t=(\xi+\eta-\tau) / 2$. Note that for each fixed $(\xi, \tau)$ in the range of integration

$$
\lim _{\eta \rightarrow 0}(\sin \eta)^{2 \alpha+1} g(\xi, \eta ; s, t)=0
$$

and that

$$
2(\sin \xi)(\sin \eta) \leq 2(\sin s)(\sin t)=\cos \tau-\cos (\xi+\eta)
$$

so that

$$
\begin{aligned}
(\sin \eta)^{2 \alpha+1}|g(\xi, \eta ; s, t)| & \leq 4 M_{\alpha}^{\prime} U_{0}^{-\alpha-1 / 2}[2(\sin s)(\sin t)]^{\alpha-1 / 2} \\
& \leq 4 M_{\alpha}^{\prime} U_{0}^{-\alpha-1 / 2}(\cos \tau-\cos \xi)^{\alpha-1 / 2} .
\end{aligned}
$$

With the substitutions $u=\cos \xi$ and $v=\cos \tau$,

$$
\begin{aligned}
\int_{\theta-\phi}^{\theta+\phi} & \int_{\theta-\phi}^{\xi}(\cos \tau-\cos \xi)^{\alpha-1 / 2} d \tau d \xi \\
& =\int_{X_{0}}^{Y_{0}} \int_{u}^{Y_{0}}(v-u)^{\alpha-1 / 2}\left(1-u^{2}\right)^{-1 / 2}\left(1-v^{2}\right)^{-1 / 2} d v d u \\
& \leq \int_{X_{0}}^{Y_{0}} \int_{u}^{Y_{0}}(v-u)^{\alpha-1 / 2}\left(Y_{0}-u\right)^{-1 / 2}\left(Y_{0}-v\right)^{-1 / 2} d v d u \\
& =\frac{B(\alpha+1 / 2,1 / 2)}{\alpha+1 / 2} U_{0}^{\alpha+1 / 2} .
\end{aligned}
$$

So

$$
\int_{\theta-\phi}^{\theta+\phi}\left|I_{1}(\xi, \eta)\right| d \xi \leq M_{\alpha}^{\prime} B(\alpha+1 / 2,1 / 2) /(2 \alpha+1)
$$

and Lebesgue's Dominated Convergence Theorem can be invoked to assert that

$$
\lim _{\eta \rightarrow 0} \int_{\theta-\phi}^{\theta+\phi} I_{1}(\xi, \eta) d \xi=0 .
$$

A similar analysis can be applied to $I_{2}(\xi, \eta)$. With $s=(\sigma+\tau) / 2, t=(\sigma-\tau) / 2$

$$
I_{3}(\xi, \eta)=-\frac{1}{4} \int_{\xi+\eta}^{\theta+\phi} \int_{\theta-\phi}^{\xi-\eta} D_{\eta}^{\alpha}(\xi, s, t) Q(s, t) R_{q}^{\alpha}(s, t ; \theta, \phi) d \tau d \sigma .
$$

The integrand is dominated, independently of $\eta$, by

$$
H(\xi ; s, t ; \theta, \phi)=\mathscr{D}^{\alpha}(\xi, s, t)\left|Q(s, t) R_{q}^{\alpha}(s, t ; \theta, \phi)\right| .
$$

We begin by showing that there is $A$, independent of $\theta$ and $\phi$, such that

$$
J(\theta, \phi)=\int_{\theta-\phi}^{\theta+\phi} \int_{\xi}^{\theta+\phi} \int_{\theta-\phi}^{\xi} H(\xi ; s, t ; \theta, \phi) d \tau d \sigma d \xi \leq A .
$$


A change in the order of integration and use of equation (2.16) shows

$$
J(\theta, \phi) \leq \mathscr{B}_{\alpha} \int_{\theta-\phi}^{\theta+\phi}\left\{\int_{\theta-\phi}^{\sigma}\left|Q(s, t) R_{q}^{\alpha}(s, t ; \theta, \phi)\right| d \tau\right\} d \sigma .
$$

Now by Lemma 3.3, and with $M_{\alpha}^{\prime \prime}=C M_{\alpha} \exp \left[K_{\alpha} H_{\alpha}(0, \pi / 2)\right]$,

$$
\begin{aligned}
& \left|Q(s, t) R_{q}^{\alpha}(s, t ; \theta, \phi)\right| \\
& \quad \leq M_{\alpha}^{\prime \prime}\left(\frac{1}{\sin s}+\frac{1}{\sin t}\right)\left(\frac{\sin s \sin t}{\sin \theta \sin \phi}\right)^{\alpha+1 / 2} \\
& \quad \leq 2 M_{\alpha}^{\prime \prime} U_{0}^{-\alpha-1 / 2}(\sin s+\sin t)[2(\sin s)(\sin t)]^{\alpha-1 / 2} .
\end{aligned}
$$

Thus

$$
\begin{aligned}
J(\theta, \phi) \leq 4 M_{\alpha}^{\prime \prime} \mathscr{B}_{\alpha} U_{0}^{-\alpha-1 / 2} \int_{\theta-\phi}^{\theta+\phi}\left\{\int_{\theta-\phi}^{\sigma}\left(\sin \frac{\sigma}{2}\right)\left(\cos \frac{\tau}{2}\right)\right. \\
\left.\times(\cos \tau-\cos \sigma)^{\alpha-1 / 2} d \tau\right\} d \sigma
\end{aligned}
$$

In the integral, make the substitutions $u=\cos \sigma / 2, v=\cos \tau / 2, a=\cos \frac{\theta+\phi}{2}$, and $b=\cos \frac{\theta-\phi}{2}$, so $U_{0}=2\left(b^{2}-a^{2}\right)$ and hence

$$
J(\theta, \phi) \leq 4 M_{\alpha}^{\prime \prime} \mathscr{B}_{\alpha} K(\theta, \phi)
$$

where

$$
K(\theta, \phi)=\left(b^{2}-a^{2}\right)^{-\alpha-1 / 2} \int_{a}^{b}\left\{\int_{u}^{b}\left(v^{2}-u^{2}\right)^{\alpha-1 / 2}\left(1-v^{2}\right)^{-1 / 2} 2 v d v\right\} d u
$$

The estimate

$$
\begin{aligned}
K(\theta, & \phi) \leq\left(b^{2}-a^{2}\right)^{-\alpha-1 / 2} \int_{a}^{b}\left\{\int_{u^{2}}^{b^{2}}\left(w-u^{2}\right)^{\alpha-1 / 2}\left(b^{2}-w\right)^{-1 / 2} d w\right\} d u \\
& =\left(b^{2}-a^{2}\right)^{-\alpha-1 / 2} B(\alpha+1 / 2,1 / 2) \int_{a}^{b}\left(b^{2}-u^{2}\right)^{\alpha} d u \\
& \leq\left(b^{2}-a^{2}\right)^{-\alpha-1 / 2} B(\alpha+1 / 2,1 / 2) \frac{1}{2} \int_{a^{2}}^{b^{2}}\left(b^{2}-w\right)^{\alpha}\left(w-a^{2}\right)^{-1 / 2} d w \\
& =\frac{1}{2} B(\alpha+1 / 2,1 / 2) B(\alpha+1,1 / 2)=\pi /(2 \alpha+1)
\end{aligned}
$$

then yields equation (3.13) with $A=4 M_{\alpha}^{\prime \prime} \mathscr{B}_{\alpha} \pi /(2 \alpha+1)$.

Fubini's Theorem and equation (3.13) guarantee the convergence of $I_{3}(\xi, \eta)$ to the integral in equation (3.12) for almost every $\xi$, and (3.13) together with Lebesgue's Dominated Convergence Theorem then yields equation (1.11).

Moreover, from equation (3.12) and the estimate above we obtain the bound

$$
\int_{0}^{\pi}\left|D_{q}^{\alpha}(\xi, \theta, \phi)\right| d \xi \leq 1+M_{\alpha}^{\prime \prime} \pi /(\alpha+1 / 2) .
$$

Finally, to obtain (iv) observe that if $q$ is nonincreasing on $(0, \pi / 2)$, then $Q(s, t)=q(s)-q(t) \leq 0$ on $\Omega, R_{q}^{\alpha}(s, t ; \theta, \phi) \geq 0$ by Lemma 3.3(ii), and $D^{\alpha}(\xi, \theta, \phi) \geq 0$, so equation (3.12) implies $D_{q}^{\alpha}(\xi, \theta, \phi) \geq 0$.

The proof of part (iii) is deferred to $\S 4$. 


\section{Convolutions, AND THE HARMONIC ANALYSIS OF CERTAIN JACOBI TYPE HYPERGROUPS}

Theorem 1.2 suggests the existence of a convolution for $L^{1}[(0, \pi), d \theta]$. The reason for this is that if we define

$$
h(\xi)=\int_{0}^{\pi} \int_{0}^{\pi} D_{q}^{\alpha}(\xi, \theta, \phi) f(\theta) g(\phi) d \theta d \phi
$$

and

$$
U_{k}(f)=\int_{0}^{\pi} u_{q, k}^{\alpha}(\theta) f(\theta) d \theta,
$$

then $\|h\|_{1} \leq A_{q}^{\alpha}\|f\|_{1}\|g\|_{1}$ and $U_{k}(h)=U_{k}(f) U_{k}(g)$.

Since in general $A_{q}^{\alpha}>1, L^{1}[(0, \pi), d \theta]$ does not become a Banach algebra with the multiplication above, thus it is necessary to renormalize. Notice that $u_{q, 0}^{\alpha}$ is positive on $[0, \pi]$ and let $m_{q}^{\alpha}$ be the measure defined by

$$
d m_{q}^{\alpha}(\xi)=\left[u_{q, 0}^{\alpha}(\xi)\right]^{2} \sin ^{2 \alpha+1} \xi d \xi,
$$

and define

$$
\begin{gathered}
K(\xi, \theta, \phi)=K_{q}^{\alpha}(\xi, \theta, \phi)=\frac{D_{q}^{\alpha}(\xi, \theta, \phi)}{u_{q, 0}^{\alpha}(\xi) u_{q, 0}^{\alpha}(\theta) u_{q, 0}^{\alpha}(\phi) \sin ^{2 \alpha+1}(\xi)}, \\
R_{q, k}^{\alpha}(\xi)=\frac{u_{q, k}^{\alpha}(\xi)}{u_{q, 0}^{\alpha}(\xi)}
\end{gathered}
$$

then we have from equation (1.11)

$$
\int_{0}^{\pi} K_{q}^{\alpha}(\xi, \theta, \phi) R_{q, k}^{\alpha}(\xi) d m_{q}^{\alpha}(\xi)=R_{q, k}^{\alpha}(\theta) R_{q, k}^{\alpha}(\phi)
$$

and setting $k=0$ in equation (4.1)

$$
\int_{0}^{\pi} K_{q}^{\alpha}(\xi, \theta, \phi) d m_{q}^{\alpha}(\xi)=1
$$

and $K_{q}^{\alpha}(\xi, \theta, \phi)$ is zero unless $|\theta-\phi| \leq \xi \leq \pi-|\theta+\phi-\pi|$. Let $L^{p}=$ $L^{p}\left[(0, \pi), d m_{q}^{\alpha}\right]$ and let $\|\cdot\|_{p}$ denote the norm in this space. Define

$$
\begin{gathered}
f^{\sim}(k)=\int_{0}^{\pi} f(\theta) R_{q, k}^{\alpha}(\theta) d m_{q}^{\alpha}(\theta) \quad\left(k \in N_{0}, f \in L^{1}\right), \\
(f * g)(\xi)=\int_{0}^{\pi} \int_{0}^{\pi} K_{q}^{\alpha}(\xi, \theta, \phi) f(\theta) g(\phi) d m_{q}^{\alpha}(\theta) d m_{q}^{\alpha}(\phi) \quad\left(f, g \in L^{1}\right) .
\end{gathered}
$$

4.1. Theorem. Let $q$ be admissible and nonincreasing on $(0, \pi / 2)$, then

(i) $L^{1}$ is a Banach algebra with respect to the operation *.

(ii) If $f$ and $g$ are nonnegative, so is $f * g$.

(iii) If $f$ and $g$ belong to $L^{1}$ and $k \in N_{0}$, then

$$
(f * g)^{\wedge}(k)=f^{\wedge}(k) g^{\wedge}(k) .
$$

For the sake of completeness, we also include 
4.2. Theorem. For general admissible $q$,

(i) * is a bounded operation on $L^{1}$, in particular

$$
\|f * g\|_{1} \leq C_{q}^{\alpha}\|f\|_{1}\|g\|_{1}
$$

with $C_{q}^{\alpha}=A_{q}^{\alpha}\left\|u_{q, 0}^{\alpha}\right\|_{\infty}\left\|1 / u_{q, 0}^{\alpha}\right\|_{\infty}^{2}$ where $A_{q}^{\alpha}$ is as in Theorem 1.2.

(ii) If $f$ and $g$ belong to $L^{1}$ and $k \in N_{0}$, then $(f * g)^{\wedge}(k)=f^{\wedge}(k) g^{\wedge}(k)$. Proof of Theorems 4.1, 4.2. Part (iii) is a consequence of equation (4.1) and Fubini's Theorem. Part (ii) holds because the nonnegativity of $D_{q}^{\alpha}$ implies the same for $K_{q}^{\alpha}$.

The nonnegativity of $K_{q}^{\alpha}$ and equation (4.2) imply that $\|f * g\|_{1} \leq\|f\|_{1}\|g\|_{1}$.

Lemma 1.1 implies that $\left\{R_{q, k}^{\alpha}\right\}_{k=0}^{\infty}$ is a complete orthogonal system in $L^{2}$, so the mapping $f \rightarrow f^{\wedge}$ is one-to-one. Thus $*$ is commutative since $(f * g) \wedge(k)=$ $f^{\wedge}(k) g^{\wedge}(k)=(g * f)^{\wedge}(k)$, and a similar argument can be used to show that $*$ is associative.

The proof of Theorem 4.2 differs from the preceding only in the lack of positivity. In this case we have by Fubini's Theorem

$$
\|f * g\|_{1} \leq \int_{0}^{\pi} \int_{0}^{\pi}|f(\theta) \| g(\phi)| \int_{0}^{\pi} \frac{\left|D_{q}^{\alpha}(\xi, \theta, \phi)\right| u_{q, 0}^{\alpha}(\xi)}{u_{q, 0}^{\alpha}(\theta) u_{q, 0}^{\alpha}(\phi)} d \xi d m_{q}^{\alpha}(\theta) d m_{q}^{\alpha}(\phi)
$$

and the result follows.

Proof of Theorem 1.2(iii). Let

$$
I(k)=\int_{0}^{\pi}\left[K_{q}^{\alpha}(\xi, \theta, \phi)-K_{q}^{\alpha}(\xi, \phi, \theta)\right] R_{q, k}^{\alpha}(\xi) d m_{q}^{\alpha}(\xi) ;
$$

then equation (4.1) implies $I(k)=0$ for all $k \in N_{0}$, so Lemma 1.1 implies

$$
K_{q}^{\alpha}(\xi, \theta, \phi)=K_{q}^{\alpha}(\xi, \phi, \theta) .
$$

Similarly, application of Fubini's Theorem to the second term of

$$
I(j, k)=\int_{0}^{\pi} \int_{0}^{\pi}\left[K_{q}^{\alpha}(\xi, \theta, \phi)-K_{q}^{\alpha}(\theta, \xi, \phi)\right] R_{q, j}^{\alpha}(\xi) R_{q, k}^{\alpha}(\theta) d m_{q}^{\alpha}(\xi) d m_{q}^{\alpha}(\theta)
$$

shows $I(j, k)=0$ for all $j, k$. Lemma 1.1 implies $\left\{R_{q, j}^{\alpha}(\xi) R_{q, k}^{\alpha}(\theta): j, k \in\right.$ $\left.N_{0}\right\}$ is a complete orthogonal family in $L^{2}\left[(0, \pi) \times(0, \pi), d m_{q}^{\alpha}(\xi) d m_{q}^{\alpha}(\theta)\right]$ (for instance, monomials $\xi^{j} \theta^{k}$ are in the closure, hence so are all polynomials in $(\xi, \theta)$, or by the Plancherel Theorem) and the full symmetry follows.

From now on, we assume $K_{q}^{\alpha}$ is nonnegative. There is a natural extension of * to a convolution of measures so that $M=M[0, \pi]$ becomes a hypergroup with respect to $*$. It is enough to prescribe the behavior of $*$ on point masses, so define $\delta_{\theta} * \delta_{\phi}$ for $0 \leq \theta, \phi \leq \pi$ by

$$
\delta_{0} * \delta_{\theta}=\delta_{\theta}, \quad \delta_{\pi} * \delta_{\theta}=\delta_{\pi-\theta} \quad(0 \leq \theta \leq \pi)
$$

and

$$
d\left(\delta_{\theta} * \delta_{\phi}\right)(\xi)=K_{q}^{\alpha}(\xi, \theta, \phi) d m_{q}^{\alpha}(\xi) \quad(0<\theta, \phi<\pi) .
$$

The definition is extended to all of $M$ by defining

$$
\int_{0}^{\pi} f(\xi) d(\mu * \nu)(\xi)=\int_{0}^{\pi} \int_{0}^{\pi} \int_{0}^{\pi} f(\xi) d\left(\delta_{\theta} * \delta_{\phi}\right)(\xi) d \mu(\theta) d \nu(\phi)
$$

for $f \in C[0, \pi], \mu, \nu \in M$. 
4.3. Theorem. ( $[0, \pi], *)$ is a hypergroup of Jacobi type $(\alpha, \alpha)$ (see [6]) with Hermitian characters $\left\{R_{q, k}^{\alpha}\right\}_{k=0}^{\infty}$ and Haar measure $m_{q}^{\alpha}$.

As usual, we define

$$
\mu^{\wedge}(k)=\int_{0}^{\pi} R_{q, k}^{\alpha}(\xi) d \mu(\xi) \quad\left(k \in N_{0}\right) .
$$

Since we have so much information from [6] and above about the characters and the product formula, we can obtain some detailed results about $R_{q, k}^{\alpha}$ and $\mu^{\wedge}$. It will also be useful to define the subspace

$$
M^{\prime}=\{\mu \in M: \mu(\{0\})=\mu(\{\pi\})=0\} .
$$

4.4. Lemma. (i) $\left|R_{q, k}^{\alpha}(\theta)\right|<1 \quad(0<\theta<\pi, k \in N)$.

(ii) $\lim _{k \rightarrow \infty} R_{q, k}^{\alpha}(\theta)=0 \quad(0<\theta<\pi)$.

(iii) $\lim _{k \rightarrow \infty} \mu^{\wedge}(k)=0 \quad\left(\mu \in M^{\prime}\right)$.

(iv) $\lim _{k \rightarrow \infty} \mu^{\wedge}(2 k)=\mu(\{0\})+\mu(\{\pi\}) \quad(\mu \in M), \lim _{k \rightarrow \infty} \mu^{\wedge}(2 k+1)=$ $\mu(\{0\})-\mu(\{\pi\})(\mu \in M)$.

Proof. (i) Setting $\phi=\theta$ in equation (4.1) we have

$$
\left[R_{q, k}^{\alpha}(\theta)\right]^{2}=\int_{0}^{\pi} K_{q}^{\alpha}(\xi, \theta, \theta) R_{q, k}^{\alpha}(\xi) d m_{q}^{\alpha}(\xi) .
$$

Since by Theorem 4.3, $R_{q, k}^{\alpha}$ are characters of a hypergroup

$$
\left|R_{q, k}^{\alpha}(\theta)\right| \leq 1 \quad(0 \leq \theta \leq \pi) .
$$

Now suppose for some $\theta \in(0, \pi)\left|R_{q, k}^{\alpha}(\theta)\right|=1$. Then from equation (3.12), $K_{q}^{\alpha}(\xi, \theta, \theta)$ is a positive continuous function on $(0,2 \theta)$. This observation combined with equations (4.2) and (4.5) show that equation (4.6) implies

$$
R_{q, k}^{\alpha}(\xi)=1
$$

for $0 \leq \xi \leq 2 \theta$. The argument can be repeated to show that equation (4.7) holds for $\xi$ in $[0,4 \theta],[0,8 \theta]$, etc., so finally $R_{q, k}^{\alpha} \equiv 1$, hence $k=0$ and (i) is proved.

(ii) follows from the asymptotic estimates (see [6, Theorem 4.1])

$$
R_{q, k}^{\alpha}(\xi)=\frac{2^{\alpha} \Gamma(\alpha+1)}{u_{q, 0}^{\alpha}(\xi)}\left(\frac{\xi}{\sin \xi}\right)^{\alpha+1 / 2} \frac{J_{\alpha}\left(m_{k} \xi\right)}{\left(m_{k} \xi\right)^{\alpha}}+I_{k}(\xi)
$$

where $m_{k}$ is a sequence of numbers such that $m_{k} / k \rightarrow 1$ as $k \rightarrow \infty$,

$$
\left|I_{k}(\xi)\right| \leq C m_{k}^{-1} \quad\left(0 \leq \sin \xi \leq m_{k}^{-1}\right),
$$

and

$$
\left|I_{k}(\xi)\right| \leq C m_{k}^{-1}\left(m_{k} \sin \xi\right)^{-\alpha-1 / 2} \log m_{k} \quad\left(m_{k}^{-1} \leq \sin \xi\right) .
$$

Relation (iii) follows directly from (i), (ii) and the Lebesgue Dominated Convergence Theorem.

Finally, to obtain (iv) note that if $\mu \in M$, then

$$
\nu=\mu-\mu(\{0\}) \delta_{0}-\mu(\{\pi\}) \delta_{\pi} \in M^{\prime}
$$

and

$$
\begin{aligned}
\mu^{\frown}(k) & =\nu^{\frown}(k)+\mu(\{0\}) R_{q, k}^{\alpha}(0)+\mu(\{\pi\}) R_{q, k}^{\alpha}(\pi) \\
& =\nu^{\frown}(k)+\mu(\{0\})+(-1)^{k} \mu(\{\pi\}) .
\end{aligned}
$$

The fact that $\delta_{\theta} * \delta_{\phi}$ is usually absolutely continuous, allows us to obtain detailed information about the harmonic analysis of $M$. 
4.5. Lemma. If $\mu, \nu \in M^{\prime}$, then $\mu * \nu$ is absolutely continuous.

Proof. If $\mu, \nu \in M^{\prime}$, then equations (4.3) and (4.4) yield

$$
\int_{0}^{\pi} f(\xi) d(\mu * \nu)(\xi)=\int_{0}^{\pi} \int_{0}^{\pi} \int_{0}^{\pi} f(\xi) K_{q}^{\alpha}(\xi, \theta, \phi) d m_{q}^{\alpha}(\xi) d \mu(\theta) d \mu(\phi) .
$$

A simple argument based on Lusin's Theorem [27, Theorem 2.23 and Example 3.18] shows that $(\mu * \nu)(E)=0$ if $E$ is a set of zero Lebesgue measure.

We are now in a position to use the methods of [8 and 28] to generalize [8, Theorems 5c, d].

4.6. Theorem. (i) The set of nontrivial complex homomorphisms of $M$ consists of $\left\{h_{k}: k=0,1,2, \ldots\right\}$ where $h_{k}(\mu)=\mu^{\wedge}(k)$ together with $h_{+}$and $h_{-}$ defined by $h_{+}(\mu)=\mu(\{0\})+\mu(\{\pi\}), h_{-}(\mu)=\mu(\{0\})-\mu(\{\pi\})$.

4.7. Theorem. A measure $\mu$ is idempotent, that is, $\mu * \mu=\mu$, if and only if

$$
\mu=\nu+\varepsilon_{0} \delta_{0}+\varepsilon_{\pi} \delta_{\pi}
$$

where $\nu$ is a finite sum of the form

$$
d \nu(\xi)=\sum_{n=1}^{N} R_{q, k_{n}}^{\alpha}(\xi)\left\|R_{q, k_{n}}^{\alpha}\right\|_{2}^{-2} d m_{q}^{\alpha}(\xi)
$$

for some strictly increasing sequence of integers $\left\{k_{n}\right\}$ and one of the following holds:

$$
\varepsilon_{0}=1, \quad \varepsilon_{\pi}=0 \text { or } \varepsilon_{0}= \pm \varepsilon_{\pi}=1 / 2 .
$$

Equivalently, (cf. [8, p. 257]), given a sequence $\left\{c_{k}\right\}_{k=0}^{\infty}, c_{k}=\mu^{\wedge}(k)$ for some idempotent measure $\mu$ if and only if for all $k, c_{k} \in\{0,1\}$, and $\left\{c_{k}\right\}_{k=0}^{\infty}$ is a sequence of period 2 with finitely many exceptions.

\section{REFERENCES}

1. R. Askey, Orthogonal polynomials and special functions, SIAM, Philadelphia, Pa., 1975.

2. B. L. J. Braaksma and H. S. V. DeSnoo, Generalized translation operators associated with a singular differential operator, Proc. Conf. Ordinary and Partial Differential Equations, Dundee 1974 (B. D. Sleeman and I. M. Michael, eds.), Lecture Notes in Math., vol. 415, Springer-Verlag, Berlin, 1974, pp. 62-77.

3. H. Chébli, Sur la positivité des opérateurs de "translation généralisée" associés à un opérateur de Sturm-Liouville sur ]0, $\infty$ [ , C. R. Acad. Sci. Paris 275 (1972), 601-604.

4. E. A. Coddington and N. Levinson, Theory of ordinary differential equations, McGraw-Hill, New York, 1955.

5. W. C. Connett, C. Markett and A. L. Schwartz, Product formulas and convolutions for angular and radial spheroidal wave functions, Trans. Amer. Math. Soc. (to appear).

6. W. C. Connett and A. L. Schwartz, Analysis of a class of probability preserving measure algebras on compact intervals, Trans. Amer. Math. Soc. 320 (1990), 371-393.

7. J. Delsarte, Sur une extension de la formule de Taylor, J. Math. Pures Appl. Sér. 917 (1938), 213-231.

8. C. F. Dunkl, Operators and harmonic analysis on the sphere, Trans. Amer. Math. Soc. 125 (1966), 250-263.

9. __ The measure algebra of a locally compact hypergroup, Trans. Amer. Math. Soc. 179 (1973), 331-348.

10. P. R. Garabedian, Partial differential equations, Wiley, New York, 1964. 
11. G. Gasper, Positivity and the convolution structure for Jacobi series, Ann. of Math. (2) 93 (1971), 112-118.

12. _ Banach algebras for Jacobi series and positivity of a kernel, Ann. of Math. (2) 95 (1972), 261-280.

13. I. I. Hirschman, Harmonic analysis and ultraspherical polynomials, Symposium on Harmonic Analysis and Related Integral Transforms, Cornell Univ., 1956.

14. V. Hutson and J. S. Pym, Generalized translation operators associated with a differential operator, Proc. London Math. Soc. (3) 24 (1972), 548-576.

15. R. I. Jewett, Spaces with an abstract convolution of measures, Adv. in Math. 18 (1975), 1-101.

16. T. H. Koornwinder, The addition formula for Jacobi polynomials and spherical harmonics, I. Summary of results, Indag. Math. 34 (1972), 188-191.

17. N. Leblanc, Classification des algèbres de Banach associêes aux opérateurs différentiels de Sturm-Liouville, J. Funct. Anal. 2 (1968), 52-72.

18. B. M. Levitan, Generalized translation operators, Israel Program for Scientific Translations, Jerusalem, 1964.

19. C. Markett, Product formulas and convolution for Fourier-Bessel series, Constructive Approximation (to appear).

20. __ A new proof of Watson's product formula for Laguerre polynomials via a Cauchy problem associated with a singular differential operator, SIAM J. Math. Anal. 17 (1986), 1010-1031.

21. __ Norm estimates for generalized translation operators associated with a singular differential operator, Indag. Math. 46 (1984), 299-313.

22. __ Product formulas for Bessel, Whittaker, and Jacobi functions via the solution of an associated Cauchy problem, Functional Analysis and Approximation (Proc. Conf. Oberwolfach 1983, P. L. Butzer, R. L. Stens, and B. Sz.-Nagy, eds.), ISNM 65, Birkhäuser, Basel, 1984, pp. 449-462.

23. __ Product formulas for eigenfunctions of singular Sturm-Liouville equations, Approximation Theory V (Proc. Conf. College Station 1986, C. K. Chui, L. L. Schumaker, and J. D. Ward, eds.), Academic Press, New York, pp. 467-470.

24. E. Michael, Topologies on spaces of subsets, Trans. Amer. Math. Soc. 71 (1951), 152-182.

25. A. Povzner, On differential equations of Sturm-Liouville type on a half axis, Amer. Math. Soc. Transl. 5 (1950), 24-101.

26. B. Riemann, Über die Fortpflanzung ebener Luftwellen von endlicher Schwingungweite, Abh. Königl. Gesellschaft der Wiss. Gottingen 8 (1860). In: Ges. Math. Werke, Leipzig, 1876, pp. 145-164.

27. W. Rudin, Real and complex analysis, McGraw-Hill, New York, 1966.

28. A. L. Schwartz, The structure of the algebra of Hankel transforms and the algebra of HankelStieltjes transforms, Canad. J. Math. 23 (1971), 236-246.

29. K. Trimèche, Transformation intégrale de Weyl et théorème de Paley-Wiener associés à un opérateur différentiel singulier sur $(0, \infty)$, J. Math. Pures Appl. 60 (1981), 51-98.

30. G. Watson, A treatise on the theory of Bessel functions, Cambridge Univ. Press, Cambridge, 1966.

31. H. F. Weinberg, A maximum property of Cauchy's problem, Ann. of Math. (2) 64 (1956), 505-513.

(W. C. Connett and A. L. Schwartz) Department of Mathematics, University of MissouriST. Louis, ST. LouIs, Missouri 63121

E-mail address: connett@umsluma.bitnet, schwartz@arch.umsl.edu

(Clemens Markett) Lehrstuhl A für Mathematik, Rheinisch-Westfälische Technische Hochschule Aachen, Templergraben 55, D-5100 Aachen, B.R.D.

E-mail address: ly010bu@dacth11.bitnet 